\title{
Estimation of fatigue S-N curves of welded joints using advanced probabilistic approach
}

\author{
Luca D'Angelo ${ }^{1, *}$, Alain Nussbaumer ${ }^{\mathrm{a}, 2}$ \\ ${ }^{a}$ Resilient Steel Structures Laboratory (RESSLAB), Swiss Federal Institute of Technology \\ Lausanne
}

\begin{abstract}
This paper provides a new advanced probabilistic approach for reliable estimation of the fatigue characteristic S-N curves of welded joints both for constant amplitude (CA) and variable amplitude (VA) loading conditions. The presented approach, which is referred to as the ML-MCS approach, combines Maximum Likelihood method (ML) and Monte-Carlo Simulations (MCS) method to estimate true $p$-quantiles of CA and VA S-N curves by using complete experimental data-sets. The ML-MCS approach includes a linearization method for use of S-N curves in combination with linear damage accumulation rule as well as for direct comparison with current standards. Application of the ML-MCS approach on two study cases and comparison with current standards shows that the use of the ML-MCS approach may have a significant impact in re-definition of CA and VA S-N curves of current standards and in particular of the CAFL, of the S-N curve second slope and of the critical value of accumulated damage at failure. The last section of the paper provides accurate guidelines for future experimental tests needed for re-definition of current standards.
\end{abstract}

Keywords: Fatigue life, S-N curves, Maximum Likelihood, Monte-Carlo Simulations

\footnotetext{
*Corresponding author

Email addresses: luca.dangelo@outlook.com (Luca D'Angelo), alain.nussbaumer@epfl.ch (Alain Nussbaumer)

${ }^{1} \mathrm{PhD}$

${ }^{2}$ Professor
} 


\section{Nomenclature}

$\begin{array}{ll}\text { CA } & \text { Constant Amplitude } \\ \text { cdf } & \text { Cumulative density function } \\ \text { CAFL } & \text { Constant Amplitude Fatigue Limit } \\ \text { CA S-N curve } & \text { S-N curve for CA fatigue loadings } \\ d_{t} & \text { Observed damage sum at the end of the } t^{\text {th }} \text { experimental test } \\ D_{c} & \text { Critical damage sum (random variable) } \\ d_{c, .5} & \text { Characteristic value of critical damage sum, corresponding to the me- } \\ \text { EV } & \text { dian of } D_{c} \\ \text { FAT } & \text { Extreme Value probability distribution } \\ \log \mathcal{N} & \text { Fatigue strength in MPa at } 2 \cdot 10^{6} \text { cycles, according to characteristic } \\ f_{Y} & \text { S-N curve } \\ F_{Y} & \text { Log-Normal probability distribution } \\ H(\cdot) & \text { Probability density function of random variable } Y \\ \mathrm{HCF} & \text { Cumulative distribution function of random variable } Y \\ \underline{\underline{I}}(\underline{\theta}) & \text { Unit step function } \\ \log \mathcal{N} & \text { High cycle fatigue: } N \geq 5 \cdot 10^{6} \text { cycles } \\ \log (\mathrm{S}-\mathrm{N}) \text { plane } & \text { Fisher information matrix for parameter vector equal to } \underline{\theta} \\ \mathrm{LS} & \text { log-Normal probability distribution } \\ m_{0} & \text { S-N plane in which both } S \text { axis and } N \text { axis are plotted in log scale } \\ m_{1} & \text { Least Squares } \\ \mathrm{MCS} & \text { Intercept of the CA S-N curve, in the log(S-N) plane } \\ \mathrm{ML} & \text { Slope of the CA S-N curve, in the log(S-N) plane } \\ n_{f} & \text { Monte-Carlo simulations } \\ n_{r} & \text { Maximum Likelihood } \\ n L L(\underline{\theta}) & \text { Number of failures } \\ n_{t o t} & \text { Number of run-outs } \\ n_{t o t, 1} & \text { Negative log-likelihood for model parameter vector equal to } \underline{\Theta}) \\ n_{t o t, 2} & \text { Number of stress range levels (number of blocks in a blocked VA load } \\ \mathcal{N} & \text { sequence or number of levels in a VA spectrum) } \\ & \text { Number of stress range levels, for stress ranges higher than or equal to } \\ & \\ & \end{array}$




\begin{tabular}{|c|c|}
\hline pdf & Probability density function \\
\hline$P_{f}$ & Probability of failure \\
\hline$P_{f}^{t}$ & Target probability of failure \\
\hline$p$-quantile & $\begin{array}{l}\text { Referred to a S-N curve, it indicates the curve which realizes } p \cdot 100 \% \\
\text { probability of failure }\end{array}$ \\
\hline$P_{s}=1-P_{f}$ & Probability of survival \\
\hline run-out & $\begin{array}{l}\text { Number of cycles reached without failure of the specimen at the end } \\
\text { of the test, either because the time limits for the test were reached or } \\
\text { because other parts of the tested specimen failed }\end{array}$ \\
\hline$S$ & Nominal applied stress range \\
\hline$S_{c o}$ & Cut-off stress range \\
\hline$V$ & Natural logarithm of the CAFL (random variable) \\
\hline$v_{.05}$ & $\begin{array}{l}\text { Characteristic value of the log-CAFL, corresponding to } 95 \% \text { exceeding } \\
\text { probability }\end{array}$ \\
\hline $\mathrm{VA}$ & Variable Amplitude \\
\hline VAFL & Variable Amplitude Fatigue Limit \\
\hline VA S-N curve & S-N curve to for VA fatigue loadings \\
\hline$\left.\left(x_{i}, y_{i}\right)\right|_{i=1 \ldots n_{\text {tot }}}$ & CA fatigue data-set \\
\hline$\alpha$ & Confidence level \\
\hline$\delta$ & Binary variable which is equal to 1 for a failure and to 0 for a run-out \\
\hline$\Delta m=m_{1}-m_{2}$ & Slope difference, in the $\log (\mathrm{S}-\mathrm{N})$ plane \\
\hline$\epsilon$ & Error term in S-N model \\
\hline$\eta_{\text {iter }}, \eta_{\text {res }}$ & $\begin{array}{l}\text { Parameters which define the number of simulations in ML-MCS scheme } \\
\text { for estimation of VA S-N model }\end{array}$ \\
\hline$\eta_{\text {sam }}$ & $\begin{array}{l}\text { Number of simulations in MCS scheme for estimation of CA character- } \\
\text { istic S-N curves }\end{array}$ \\
\hline$\theta$ & CA S-N model vector \\
\hline$\Theta$ & VA S-N model vector \\
\hline$\mu_{D}$ & Location parameter of critical damage probability distribution, $D_{c}$ \\
\hline$\mu_{V}$ & Location parameter of log-CAFL probability distribution \\
\hline$\sigma$ & Logarithm of the scale parameter of log-life probability distribution \\
\hline$\sigma_{V}$ & Logarithm of the scale parameter of log-CAFL probability distribution \\
\hline$\sigma_{D}$ & Scale parameter of critical damage probability distribution, $D_{c}$ \\
\hline$\underline{\underline{\Sigma}}$ & VA S-N model covariance matrix \\
\hline$\underline{\rho}$ & CA S-N model correlation matrix \\
\hline
\end{tabular}


Standardized normal pdf

Load dynamic amplification factor

Standardized normal cdf

Percentage of stress range cycles of a loading spectrum, which exceeds the characteristic value of CAFL 


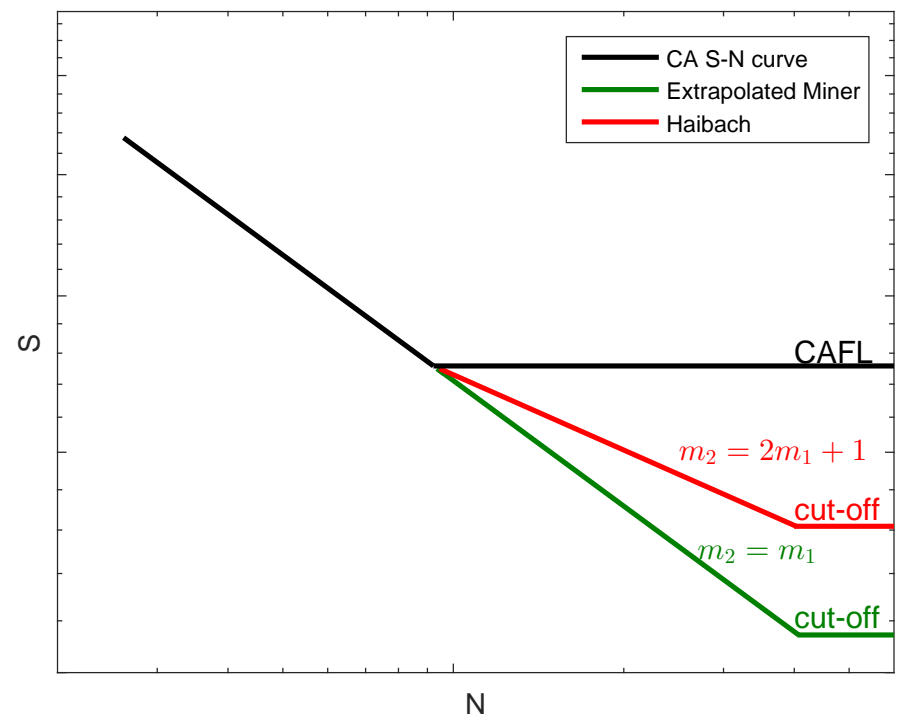

Figure 1: VA S-N curves

\section{Introduction}

Traditional fatigue analysis of welded joints under variable amplitude (VA) loadings is based on the nominal stress approach, also called classification method. The fatigue verification can be based: 1) On the constant amplitude fatigue limit (CAFL) or 2) On damage accumulation rule, having assumed an S-N curve formulation below the CAFL. The most largely used rule is the Haibach's rule [1], which considers a S-N curve with a reduced slope, $m_{2}=2 m_{1}+1$ below the CAFL (see Figure 1).

In this paper, we will focus on $\mathrm{S}-\mathrm{N}$ curves under normal stress ranges, but similar developments can also be made for shear stress ranges.

\section{1. $S$ - $N$ curves for constant amplitude loadings}

Within the CAFL-based verification, the choice of the constant amplitude (CA) fatigue S-N curve is of primary importance. CA S-N curves express fatigue strength of welded joints under CA loadings by giving the fatigue life, $N$, at each stress range, $S$, for a certain probability of survival, $P_{s}$, on the basis of a certain confidence level. S-N curves are estimated by using 
experimental fatigue test results; however, due to the inherent randomness in the fatigue life, a statistical treatment of experimental results is needed.

In Eurocode 3 Part 1-9 [2], median S-N curve $\left(P_{s}=50 \%\right)$ is derived by fitting a linear regression which has a slope $m_{1}=-3$ to the failure data points, using a least squares (LS) analysis procedure. The characteristic S-N curve is then derived by translating the median $\mathrm{S}-\mathrm{N}$ curve on the $5 \%$ prediction bound, at $2 \cdot 10^{6}$ cycles, and by arbitrarily assuming the CAFL at $5 \cdot 10^{6}$ cycles. This statistical procedure is unrealistic since it neglects important information from experimental observations by disregarding run-out data and it arbitrarily assumes the CAFL without experimental proof. For clarification, it is important to recall here that $\mathrm{S}-\mathrm{N}$ curves in Eurocode 3 Part 1-9 [2] were established for the design of new structures. Indeed, simplicity (i.e. having a set of parallel curves) was prioritized with respect to accuracy (i.e. different curve slopes, position of the CAFL). This choice was motivated by the need of ranking the different details according to their fatigue reserve (ratio between the applied nominal stress range and the fatigue strength at $2 \cdot 10^{6}$ cycles (FAT)), in order to quickly be able to identify the fatigue critical locations. Limitations above, in particular when assessing existing structures and needing more accuracy, may be overcome by using a stochastic S-N model including aleatory CAFL and by estimating model parameters with Maximum Likelihood (ML) method. Many authors used ML method to estimate S-N curves for fatigue experimental data-sets containing both failures and run-outs [3, 4]. In [5] Pascual proposed a Random Fatigue Limit (RFL) model to fit a complete experimental data-set using ML method and to estimate median and characteristic S-N curves with Profile Likelihood Ratio (PLR) based confidence bounds. In [6] Lassen et al. fitted the RFL model from Pascual to two experimental data-sets of a plate with welded attachments. In Part 3 of the JCSS Probabilistic Model Code (PMC) [7] a ML-based approach is recommended for the estimation of S-N curves. The epistemic uncertainty related to the model parameter vector is estimated by using the Fisher information matrix. However, this recommended approach is affected by the following limitations: 1) It does not specify if the CAFL has to be considered as an aleatory variable whose parameters are included in the model parameter vector or if it has to be considered as a deterministic value which is arbitrarily assumed at a given number of cycles.; and 2) It does not specify how to relate the epistemic uncertainty on the parameter fit to the aleatory uncertainty on the fatigue life.

The existence of the CAFL is still an object of dispute; Sonsino [8] pro- 
poses a CA bi-linear S-N curve where stress range cycles below the knee point are accounted with a slope $m_{1}^{\prime}=-22$. The knee point is arbitrarily fixed at $N=10^{7}$. The slope $m_{1}^{\prime}=-22$ is also arbitrarily fixed in order to have $10 \%$ constant stress range decrease with respect to the $\log (N)$ axis for each order of magnitude. This approach is presented as safe due to the lack of experimental results in the high cycle fatigue $(\mathrm{HCF})$ region $\left(N \geq 5 \cdot 10^{6}\right.$ cycles) and the related high scatter of fatigue life in this region which does not allow to provide experimental validation to the CA bi-linear S-N model. For this reason the authors believe that the choice of a CA S-N model including random CAFL seems to be the most realistic choice allowing for correct interpretation of experimental results in the HCF region.

The RFL model proposed by Pascual, allows to overcome limitations of current Eurocode approach but it does not give an explicit method to derive the true $p$ - quantile S-N curve from ML estimate of CA S-N stochastic model parameters. Furthermore, the RFL model-based median S-N curve is not linear and direct comparison with current standards is not straightforward.

In this article a new approach, based on the ML method and on the Monte-Carlo Simulations (MCS) method, is proposed for estimation of median and characteristic S-N curves under CA fatigue loadings. The presented approach is referred as ML-MCS approach.

The novel contribution of this approach allows for increasing reliability of estimated CA S-N curves and it can be summarized in following points: 1) Linear S-N model with random CAFL is fitted directly to experimental data; 2) $p$-quantiles of $\mathrm{S}-\mathrm{N}$ curve, corresponding to the true $(100 \cdot p \%)$ probability of failure, are estimated using MCS method with nested sampling of epistemic uncertainty on the parameter fit and aleatory uncertainty on the fatigue life; 3) Linearization of ML-MCS-based characteristic S-N curves allows to make direct comparison with current standards.

\section{2. $S$ - $N$ curves for variable amplitude loadings}

Within the damage accumulation-based verification, the choice of a reliable CA fatigue $\mathrm{S}-\mathrm{N}$ curve is of primary importance, but it is not sufficient, since a reliable definition of damage accumulation is also required.

The fatigue verification of welded joints under VA loadings is conventionally based on CA S-N curves, used in combination with Miner's linear damage accumulation rule. According to Miner's rule, the following condition has to 
be fulfilled for fatigue verification:

$$
\sum_{i=1}^{n_{t o t}} \frac{n_{i}}{N_{i}} \leq D_{c}
$$

where:

- $n_{i}$ is the number of cycles corresponding to the applied nominal stress range, $S_{i}$;

- $n_{\text {tot }}$ is the number of stress range levels (number of blocks in a blocked VA load sequence or number of levels in a VA spectrum);

- $N_{i}$ is the number of cycles to failure corresponding to $S_{i}$;

- $D_{c}$ is the critical value of the damage sum, often taken as unity.

The first critical aspect in using Miner's rule is how to consider the influence on the fatigue damage of the stress range cycles below the CAFL.

In Eurocode standards [9] and in IIW recommendations [10], the CA characteristic S-N curve is bent at the CAFL position from the slope $m_{1}=-3$ to the slope $m_{2}=-5$; a cut-off limit is fixed at $10^{8}$ cycles. The use of the reduced (flatter) slope $m_{2}=2 m_{1}+1=-5$ has first been proposed by Haibach [1], by assuming a continuously decreasing of the fatigue limit as a function of the linear accumulated damage [11] (see Figure 1. A cut-off limit, or Variable Amplitude Fatigue Limit (VAFL) is set at the value reached at $10^{8}$ cycles, that is $0.405 \cdot$ FAT).

In AASHTO bridge design specifications [12], the CA S-N curve is extrapolated below the CAFL with no slope change, but a variable amplitude fatigue limit (VAFL) is set at CAFL/2, which is another way of dealing with all stress ranges lower than CAFL that contribute less to fatigue damage.

Up to now, the use of the second slope $m_{2}$ has not been sufficiently justified by means of VA fatigue test results; thus the influence of the stress range cycles below the CAFL, on the fatigue damage, is still under debate $[13,14,15,16,17]$.

The second critical aspect in using Miner's rule is related to the choice of the critical value of the damage sum, $D_{c}$.

Miner and Palmgren [18] settled $D_{c}$ to 1.0 by assuming that the fatigue damage corresponding to each stress cycle of a VA loading sequence is the same as that due to the same stress cycle under CA loading sequence. However, 
many studies have shown that the stress cycles of a VA loading sequences could be more damaging than the same stress cycles under CA loading, with the result that the Miner's rule can be unsafe in some cases.

Existing studies $[16,19,13,20,21,22,23,24,15,25]$ give a qualitative indication of influence of different VA loading spectrum parameters (load sequence, stress ratio, spectrum shape, loading bandwidth and block length) on the fatigue strength of different types of welded connections; however, these works only provide general trends and do not present a rigorous statistical approach which allows to make inference about the reduced slope, $m_{2}$, and the critical damage sum, $D_{c}$. Moreover, they are affected by two substantial limitations: 1) The CA S-N curves used with Miner's rule have inaccurate definition of CAFL and of fatigue life scatter (especially in HCF region); 2) The approach used in these studies is qualitative and does not allow one to formally characterize the randomness in the S-N curve variables and in the critical damage sum variable.

In order to provide a reliable estimate of characteristic VA S-N curves a new probabilistic scheme has been developed and integrated into the MLMCS approach presented in Section 1.2. The critical damage sum is modeled as a random variable whose parameters are included in the VA S-N model parameter vector. The slope of the characteristic S-N curve and the parameters of $D_{c}$ distributions are estimated by using the new scheme which combines ML and MCS techniques by using the CA S-N stochastic model and the experimental data-set from VA fatigue tests.

The novel contribution of this scheme is summarized in following points:

- The slope of the $\mathrm{S}-\mathrm{N}$ curve below the CAFL, $m_{2}$ and the critical damage sum $D_{c}$ are validated by using VA experimental results;

- The effect of the stress range cycles below the CAFL is not arbitrarily assumed but it's represented by the estimated $m_{2}$ and $D_{c}$;

- Loading spectrum parameters (load sequence, stress ratio, spectrum shape, loading bandwidth and block length effects) are represented in the variability of the $D_{c}$ random variable. The representativeness of the estimated characteristic VA S-N curve is strictly connected to the use of realistic loading spectra having representative parameters when experimental data-sets are produced.

\subsection{Structure of the paper}

The article is structured as follows: 
- in Section 2 the ML-MCS approach for estimation of characteristic CA and VA S-N curves is presented;

- in Section 3 the application of the ML-MCS approach on two study cases is presented;

- in Section 4 results of analysis of considered study cases are presented;

- in Section 5 results are discussed;

- in Section 6 the impact of use of the presented approach for estimation of CA and VA S-N curves is discussed;

- in Section 7 areas of future works are identified and accurate guidelines for future experimental tests are provided.

\section{ML-MCS method}

\section{1. $S$ - $N$ curves for $C A$ loadings}

The following linear CA S-N stochastic model describes the relationship between the nominal applied stress range, $S$, and the number of cycles to failure, $N$, under CA loadings (see Figure 2 ):

$$
Y=\frac{m_{0}+m_{1} X}{H(X-V)}+\epsilon(0, \exp (\sigma))
$$

where: $Y$ is the natural logarithm ${ }^{3}$ of the number of cycles, $N$; $X$ is the natural logarithm of the nominal applied stress range, $S ; m_{0}$ is the intercept of the $\mathrm{S}-\mathrm{N}$ curve in the $\log (\mathrm{S}-\mathrm{N})$ plane; $m_{1}$ is the slope of the $\mathrm{S}-\mathrm{N}$ curve; $H(\cdot)$ is the unit step function; $V$ is the natural logarithm of the CAFL; and $\epsilon$ is the error term, which is assumed to be normally distributed with mean equal to 0 and standard deviation equal to $\exp (\sigma)$. From the last assumption it follows that the difference between $Y$ and its mean $E(Y)$ is normally distributed with mean equal to 0 and standard deviation equal to $\exp (\sigma)$.

$V$ is a random variable following a Normal or an Extreme Value (EV) distribution with probability density function (pdf) equal to $f_{V}\left(v, \mu_{V}, \sigma_{V}\right)=$

\footnotetext{
${ }^{3}$ Natural logarithms are preferred since they are mathematically less heavy to manipulate
} 


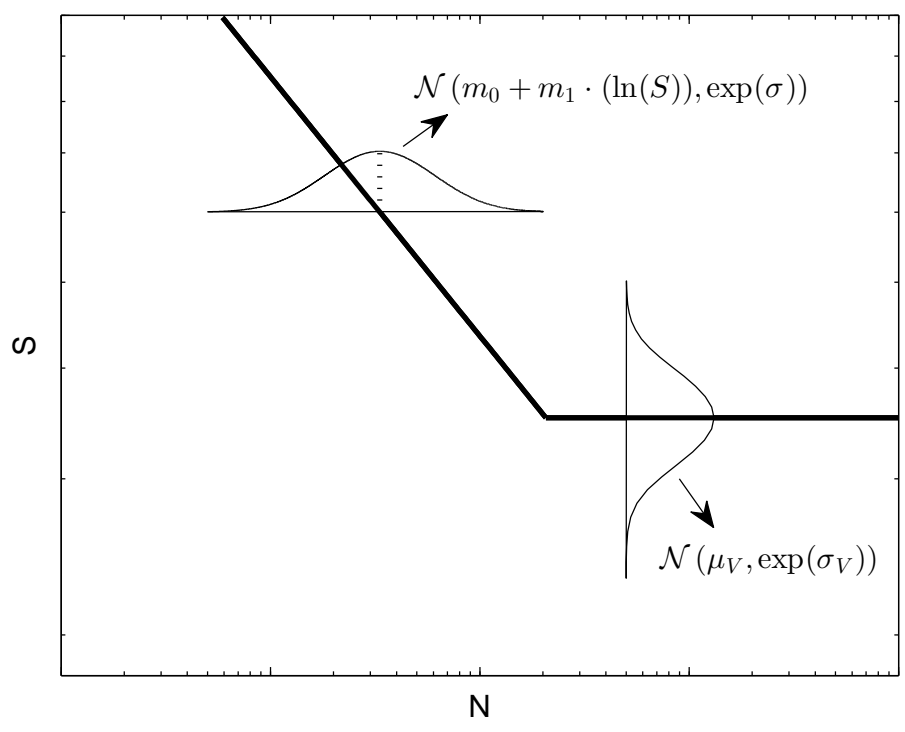

Figure 2: Median CA S-N curve with model parameters (case of $V=\mathcal{N}$ )

$\frac{1}{\exp \left(\sigma_{V}\right)} \phi_{V}\left(\frac{v-\mu_{V}}{\exp \left(\sigma_{V}\right)}\right)$, where $\phi_{V}$ is either the standardized Normal pdf or the standardized EV pdf.

The log-fatigue life, $Y$, is a random variable following a Normal distribution with pdf equal to $f_{Y}(\underline{\theta} ; y, x)=\int_{-\infty}^{x} \frac{1}{\exp \left(\sigma+\sigma_{V}\right)} \phi_{Y \mid V}(\underline{\theta} ; y, x) \phi_{V}(\underline{\theta} ; v) \mathrm{d} v$, where $\phi_{Y \mid V}$ is the standardized Normal pdf and $\theta=\left(m_{0}, m_{1}, m_{2}, \sigma, \mu_{V}, \sigma_{V}\right)$ is the CA stochastic model parameter vector. The pdf $f_{Y}$ expresses the probability of having a failure at $Y=y$ for an applied stress range equal to $\exp (x)$. The probability of having a failure at $Y \leq y$ is given by the cumulative distribution function (cdf), $F_{Y}(\underline{\theta} ; y, x)=\int_{-\infty}^{x} \frac{1}{\exp \left(\sigma_{V}\right)} \Phi_{Y \mid V}(\underline{\theta} ; y, x) \phi_{V}(\underline{\theta} ; v) \mathrm{d} v$, where $\Phi_{Y \mid V}$ is the standardized Normal cdf. Since there are no closed forms of $f_{Y}$ and $F_{Y}$, they have to be evaluated numerically.

The sample log-Likelihood is related to the pdf and the cdf of log-fatigue life Y as follows:

$$
n L L(\underline{\theta})=-\sum_{i=1}^{n_{f}} \ln \left(f_{Y}\left(\underline{\theta} ; y_{i}, x_{i}\right)\right)-\sum_{i=1}^{n_{r}} \ln \left(1-F_{Y}\left(\underline{\theta} ; y_{i}, x_{i}\right)\right)
$$

where $\left.\left(x_{i}, y_{i}\right)\right|_{i=1, \ldots, n_{t o t}}$ is the experimental data-set, $n_{f}$ is the number of failures and $n_{r}$ is the number of run-outs. The sample log-Likelihood, $L(\underline{\theta})$, 
expresses the probability of observing $\left(y_{1}, \ldots, y_{n_{\text {tot }}}\right)$, at log-stress ranges $\left(x_{1}, \ldots, x_{n_{\text {tot }}}\right)$ for a given model parameter vector, $\underline{\theta}$.

The ML estimate $\underline{\hat{\theta}}$ of $\underline{\theta}$ is the model parameter vector which minimizes the negative sample log-Likelihood, $n L L(\underline{\theta})$. Under some regularity conditions, ML estimators have asymptotic normality property [26]: as the sample size increases, the distribution of $\underline{\theta}$ tends to the Normal distribution with mean equal to $\underline{\hat{\theta}}$ and covariance matrix, $\underline{\rho}$, equal to the inverse of the observed Fisher Information matrix, $\underline{\underline{I}}(\underline{\hat{\theta}})^{-1}$.

The new ML-MCS scheme presented in this paper allows for computing the $p$-quantile, $y_{p}(\tilde{x})$, of the fatigue log-life, at the stress range, $\exp (\tilde{x})$, through following steps:

1. The $p$-value is chosen, with $P_{f}^{t}=p$.

2. The parameter $\eta_{\text {sam }}$ (sample size, typically $1 \cdot 10^{5}$ ) is chosen.

3. The sample $\left.\underline{\theta}_{i}\right|_{i=1 \ldots \eta_{\text {sam }}}$ is generated from the multivariate normal distribution $\mathcal{N}(\underline{\hat{\theta}}, \underline{\rho})$; then, $\left.y\left(\underline{\theta}_{i}, \tilde{x}\right)\right|_{i=1 \ldots \eta_{\text {sam }}},\left.v\left(\underline{\theta}_{i}\right)\right|_{i=1 \ldots \eta_{\text {sam }}}$ are sampled by using the sampled $\left.\underline{\theta}_{i}\right|_{i=1 \ldots} \eta_{\text {sam }}$. The epistemic uncertainty of S-N model parameters is taken into account in the $\left.\underline{\theta}_{i}\right|_{i=1 \ldots \eta_{s a m}}$ sampling, while the aleatory uncertainty of the CAFL and the aleatory uncertainty of the fatigue life are taken into account in the $\left.y\left(\underline{\theta}_{i}, \tilde{x}\right)\right|_{i=1 \ldots \eta_{\text {sam }}},\left.v\left(\underline{\theta}_{i}\right)\right|_{i=1 \ldots \eta_{\text {sam }}}$ sampling.

4. For each element of the sample, the probability $P_{f_{i}}\left(y_{p}(\tilde{x})\right)$ is computed:

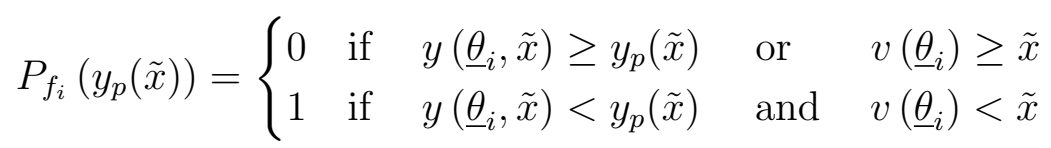

5. The objective function, $\mathcal{O}\left(y_{p}(\tilde{x})\right)$, is built:

$$
\mathcal{O}\left(y_{p}(\tilde{x})\right)=\left[\frac{\left(\sum_{i=1}^{\eta_{s a m}} P_{f_{i}}\left(y_{p}(\tilde{x})\right)\right)}{\eta_{\text {sam }}}-P_{f}^{t}\right]^{2}
$$

It is noted that the objective function expresses the square deviation of the expected value of the $\mathrm{S}-\mathrm{N}$ stochastic model-based probability of failure from the $p$-value.

6 . The $p$-quantile of the fatigue log-life, at stress range exp $(\tilde{x})$,is computed by minimizing the objective function, $\mathcal{O}\left(y_{p}(\tilde{x})\right)$ :

$$
\hat{y}_{p}(\tilde{x})=\underset{y_{p}(\tilde{x})}{\operatorname{argmin}}\left(\mathcal{O}\left(y_{p}(\tilde{x})\right)\right)
$$




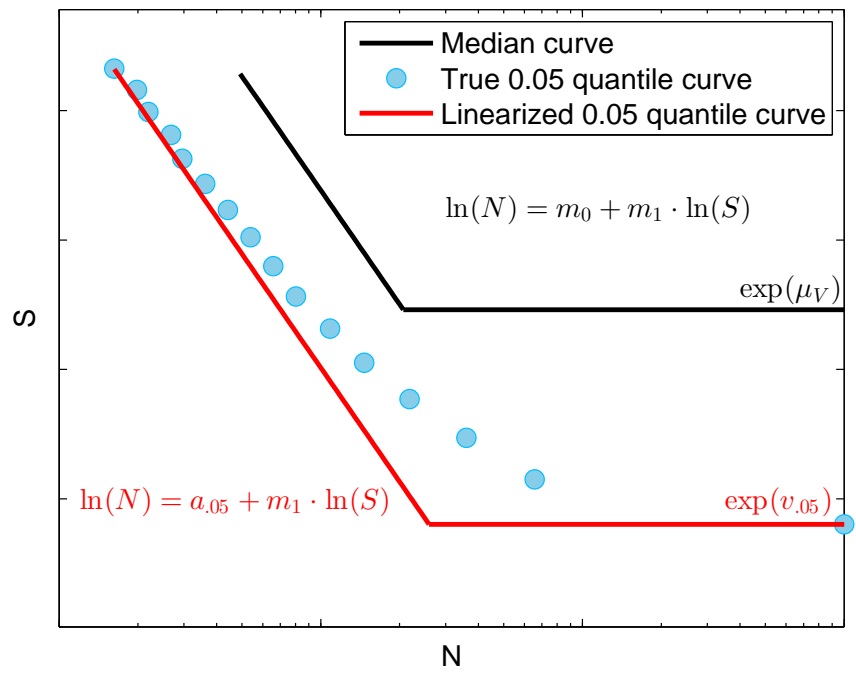

Figure 3: ML-MCS-based median, 0.05 quantile and linearized 0.05 quantile S-N curves

The 0.05 quantile S-N curve, obtained with the scheme above, represents ML-based characteristic CA S-N curve. Since ML-based characteristic S-N curve is non-linear (see Figure 3), a linearization scheme is proposed here for direct comparison with characteristic S-N curves from standards:

1. A straight line with slope equal to $\hat{m}_{1}$ (ML estimate of $m_{1}$ parameter) and passing through the true 0.05 quantile of the fatigue life at the maximum tested stress range, is drawn;

2. The line above is intersected with the horizontal line representing the true 0.05 quantile of the CAFL distribution.

The ML-MCS-based linearized characteristic S-N curve is shown in Figure 3.

\section{2. $S$ - $N$ curves for $V A$ loadings}

The S-N curve under VA loadings (Equation 4) and the fatigue failure condition (Equation 5) describe the VA S-N stochastic model:

$$
Y= \begin{cases}m_{0}+m_{1} X+\epsilon(0, \exp (\sigma)), & \text { for } X>V \\ m_{0}+V \Delta m+\left(m_{1}-\Delta m\right) X+\epsilon(0, \exp (\sigma)), & \text { for } X \leq V\end{cases}
$$




$$
\sum_{i=1}^{n_{\text {tot }}} \frac{n_{i}}{\exp \left(Y\left(x_{i}\right)\right)}=D_{c}\left(\mu_{D}, \sigma_{D}\right), \quad \text { if } \max \left(x_{i}\right)>V
$$

where $m_{0}$ and $m_{1}$ are the intercept and the slope of the S-N curve; $\epsilon(0, \exp (\sigma))$ is the error term; $V\left(\mu_{V}, \sigma_{V}\right)$ is the natural logarithm of the $\mathrm{CAFL}^{4} ; \Delta m=$ $m_{1}-m_{2}$, where $m_{2}$ is the slope of the S-N curve below the CAFL; and $D_{c}\left(\mu_{D}, \sigma_{D}\right)$ is the critical damage sum. $D_{c}$ is a random variable following a log-Normal (conventional choice) or a Weibull distribution with location parameter equal to $\mu_{D}$ and scale parameter equal to $\sigma_{D}$.

The VA S-N stochastic model is defined by the model parameter vector $\underline{\Theta}=\left.\left(\underline{\theta}, \mu_{D}, \sigma_{D}\right)\right|_{\Delta m}$ and by the covariance matrix $\underline{\underline{\Sigma}}(\underline{\Theta})$.

The generic VA fatigue data-set is denoted as follows:

$$
\left.\left(\varpi_{t}, y_{t}\right)\right|_{t=1 \ldots n_{t s}}
$$

where $\varpi_{t}$ is the $t^{\text {th }}$ stress range spectrum, $y_{t}$ is the natural logarithm of number cycles to the end of the $t^{\text {th }}$ test and $n_{t s}$ is the total number of tests. The damage, $d_{t}$, associated with the $t^{\text {th }}$ test, is:

$d_{t}(\underline{\theta}, \Delta m)=\sum_{i_{1}=1}^{n_{\text {tot }_{1}}} \frac{n_{i}}{\exp \left(Y\left(m_{0}, m_{1}, \sigma ; x_{i}\right)\right)}+\sum_{i_{2}=1}^{n_{\text {tot }_{2}}} \frac{n_{j}}{\exp \left(Y\left(m_{0}, m_{1}, \sigma, V\left(\mu_{V}, \sigma_{V}\right), \Delta m ; x_{j}\right)\right)}$

where $n_{\text {tot }_{1}}$ and $n_{\text {tot }_{2}}$ are respectively the number of stress range cycles above the CAFL and below the CAFL in the spectrum $\varpi_{t}$.

The fatigue failure condition is:

$$
d_{t}(\underline{\theta}, \Delta m)=D_{c}\left(\mu_{D}, \sigma_{D}\right)
$$

where the critical damage sum, $D_{c}$, is a random variable following a logNormal or a Weibull distribution, with parameters $\mu_{D}$ and $\sigma_{D}$.

The new ML-MCS scheme presented in this paper allows for computing parameters $\mu_{D}, \sigma_{D}$ and $\Delta m$, through following steps:

1. The experimental VA fatigue data-set is re-sampled $\eta_{\text {res }}$ time. The resampled experimental data-set is:

$$
\left.\left(\varpi_{t}, y_{t}\right)\right|_{t=1, \ldots, n_{t s} \cdot \eta_{\text {res }}}
$$

\footnotetext{
${ }^{4} m_{0}, m_{1}, \sigma, \mu_{V}, \mu_{V}$ are defined in the CA S-N stochastic model
} 
2. One accumulated damage sample $\left.d_{t}\right|_{t=1, \ldots, n_{t s} \cdot \eta_{\text {res }}}$ is generated by using the re-sampled experimental data-set $\left.\left(\varpi_{t}, y_{t}\right)\right|_{t=1, \ldots, n_{t s} \cdot \eta_{\text {res }}}$ and by sampling S-N curves from the CA S-N stochastic model. When sampling S-N curves, at each step one vector $\underline{\tilde{\theta}}_{k}$ is sampled from the CA S-N stochastic model $[\underline{\theta}, \underline{\rho}]$, then, one $\mathrm{S}-\mathrm{N}$ curve is sampled by using the sampled $\underline{\tilde{\theta}}_{k}$. The epistemic uncertainty of S-N model parameters is taken into account in the $\underline{\tilde{\theta}}_{k}$ sampling, while the aleatory uncertainty of the CAFL and the aleatory uncertainty of the fatigue life are taken into account in the S-N curve sampling. Re-sampling of the VA-fatigue data-set allows for taking into account the effect of loading spectrum parameters on the uncertainty of critical damage, $D_{c}$.

For a given value of parameter $\Delta \tilde{m}$ the negative log-Likelihood of the sample $\left.d_{t}\right|_{t=1, \ldots, n_{t s} \cdot \eta_{\text {res }}}$ is computed as follows:

$$
\begin{aligned}
\left.n L L_{k}\left(\mu_{D_{k}}, \sigma_{D_{k}}\right)\right|_{\Delta \tilde{m}}= & -\sum_{j=1}^{\eta_{\text {res }}}\left[\sum_{t=1}^{n_{f}} \ln \left(f_{D}\left(\mu_{D_{k}}, \sigma_{D_{k}}, \Delta \tilde{m} ; d_{t}\right)\right)+\right. \\
& \left.\sum_{t=1}^{n_{r}} \ln \left(1-F_{D}\left(\mu_{D_{k}}, \sigma_{D_{k}}, \Delta \tilde{m} ; d_{t}\right)\right)\right]
\end{aligned}
$$

where $n_{f}$ is the number of failures, $n_{r}$ is the number of run-outs, $f_{D}$ is the pdf of the random variable $D_{c}$ and $F_{D}$ is the cdf of the random variable $D_{c}$.

3. ML estimates of parameters $\mu_{D}$ and $\sigma_{D}$ are computed by minimizing the negative log-Likelihood of the re-simulated sample:

$$
\left(\hat{\mu}_{D}, \hat{\sigma}_{D}\right)_{k}=\arg \min _{\mu_{D_{k}}, \sigma_{D_{k}}}\left(\left.n L L_{k}\left(\mu_{D_{k}}, \sigma_{D_{k}}\right)\right|_{\Delta \tilde{m}}\right)
$$

4. Steps 1-3 are iterated $\eta_{\text {iter }}$ times.

The $\underline{\underline{G}}$ matrix and the $\underline{L}$ vector are built:

$$
\begin{array}{cc}
\underline{\underline{G}}=\left.\left[(\underline{\theta})_{k} \quad\left(\hat{\mu}_{D}\right)_{k} \quad\left(\hat{\sigma}_{D}\right)_{k}\right]\right|_{\Delta \tilde{m}} \quad \text { for } \quad k=1, \ldots, \eta_{\text {iter }} \\
\underline{L}=\left.\left[(n \hat{L} L)_{k}\right]\right|_{\Delta \tilde{m}} & \text { for } \quad k=1, \ldots, \eta_{\text {iter }}
\end{array}
$$

5. Under the assumption of asymptotic normality of ML estimators ${ }^{5} \hat{\mu}_{D}, \hat{\sigma}_{D}, n \hat{L} L$, it is observed that: 1) The matrix $\underline{\underline{G}}$ contains $n_{i t e r}$ realizations of the

\footnotetext{
${ }^{5}$ This hypothesis is verified by using probability plots
} 
multivariate Normal random variable $\left(\underline{\theta}, \hat{\mu}_{D}, \hat{\sigma}_{D}\right)$; and 2) The vector $\underline{L}$ contains $\eta_{\text {iter }}$ realizations of the Normal random variable $n \hat{L} L$.

For a given value of parameter $\Delta \tilde{m}$, the VA S-N model parameter vector, $\underline{\Theta}$, and the covariance matrix, $\underline{\underline{\Sigma}}(\underline{\Theta})$ are computed as follows:

$$
\begin{aligned}
& \underline{\Theta}(\Delta \tilde{m})=\left.E[\underline{\underline{G}}]\right|_{\Delta \tilde{m}} \\
& \underline{\underline{\underline{m}}}(\Delta \tilde{m})=\left.E\left[(\underline{\underline{G}}-E(\underline{\underline{G}})) \cdot(\underline{\underline{G}}-E(\underline{\underline{G}}))^{T}\right]\right|_{\Delta \tilde{m}}
\end{aligned}
$$

The mean of the $n \hat{L} L$ distribution is indicated as $\overline{n \hat{L} L}$.

6. Steps 1-5 are repeated for different values of $\Delta \tilde{m}$. The ML estimate of the parameter $\Delta m$ is chosen as the value which minimizes the mean of the $n \hat{L} L$ distribution ${ }^{6}$ :

$$
\Delta \hat{m}=\underset{\Delta \tilde{m}}{\operatorname{argmin}}(\overline{n \hat{L} L}(\Delta \tilde{m}))
$$

The ML estimate of the VA S-N model parameter vector and the covariance matrix are:

$$
\begin{aligned}
& \underline{\hat{\Theta}}=\underline{\Theta}(\Delta \hat{m}) \\
& \underline{\underline{\Sigma}}=\underline{\underline{\Sigma}}(\Delta \hat{m})
\end{aligned}
$$

A sensitivity study with respect to the relative variation in $\overline{n \hat{L} L}$ is performed in order to determine the parameters $\eta_{\text {iter }}$ and $\eta_{\text {res }}$ that have to be used in the ML-MCS scheme described above. Since the $\overline{n \hat{L} L}$ is not a deterministic parameter, the minimum search algorithm includes a Box-plot test to ensure that $\overline{n \hat{L} L}(\Delta \hat{m})$ is lower than $\overline{n \hat{L} L}(\Delta \hat{m} \pm 1)$ at $5 \%$ significance level. The ML-MCS based characteristic S-N curve is defined as follows:

$$
y_{.05}= \begin{cases}a_{.05}+\hat{m}_{1} x, & \text { for } x>v_{.05} \\ a_{.05}+v_{.05} \Delta \hat{m}+\left(\hat{m}_{1}-\Delta \hat{m}\right) x, & \text { for } x \leq v_{.05}\end{cases}
$$

The characteristic S-N curve in Equation 10 has to be used in combination

\footnotetext{
${ }^{6}$ The choice of computing different $\Theta(\Delta \tilde{m})$ vectors by assuming different values of $\Delta \tilde{m}$ (steps 1-5) and then to proceed in searching for the value $\Delta \hat{m}$ which minimizes $\overline{n \hat{L} L}(\Delta \hat{m})$ (step 6) was made because the initial choice of including $\Delta m$ in the model parameter vector did not work in terms of numerical optimization
} 


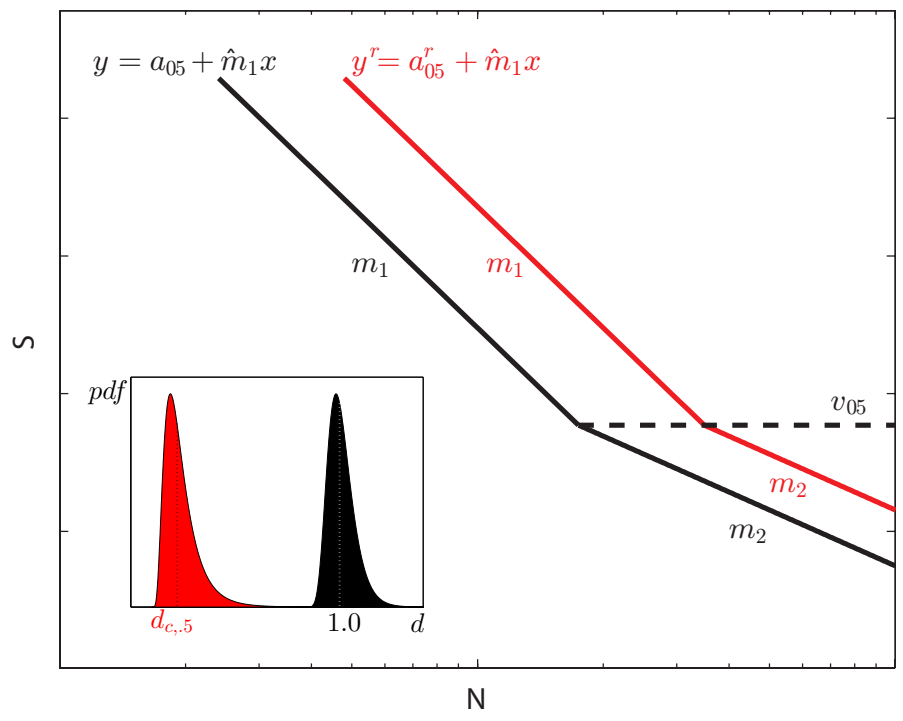

Figure 4: ML-MCS-based VA characteristic S-N curve and re-scaled VA characteristic S-N curve

with following damage accumulation rule:

$$
\sum_{i=1}^{n_{t o t}} \frac{n_{i}}{\exp \left(y_{.05}\left(x_{i}\right)\right)} \leq d_{c, .5}, \quad \text { with } d_{c, .5}=F_{D}^{-1}\left(0.5, \mu_{D}, \sigma_{D}\right)
$$

where $F_{D}^{-1}$ is the inverse of the cdf of the critical damage, $D_{c}$.

The ML-MCS-based VA characteristic S-N curve has to be re-scaled for direct comparison with VA characteristic S-N curves from the standards, which consider $d_{c, .5}=1.0$.

The ML-MCS-based re-scaled VA characteristic S-N curve (see Figure 4) is defined as follows:

$$
y_{.05}^{r}= \begin{cases}a_{.05}-\ln \left(1 / d_{c, .5}\right)+\hat{m}_{1} x, & \text { for } x>v_{.05} \\ a_{.05}-\ln \left(1 / d_{c, .5}\right)+v_{.05} \Delta \hat{m}+\left(\hat{m}_{1}-\Delta \hat{m}\right) x, & \text { for } x \leq v_{.05}\end{cases}
$$

Three cases are considered for the cut-off stress range, $\left.S_{c o}: 1\right)$ No cut-off stress range; 2) Cut-off stress range at 14.6 MPa, which corresponds to the cut-off stress range of the lowest FAT category in EN 1993-1-9; 3) Cut-off stress range at $50 \%$ of the CAFL, according to $[12,27]$. 


\section{Study cases}

The CA S-N stochastic model is fitted to two different fatigue data-sets:

1. From CA fatigue testing of 29 welded in-plane gussets having attachment length, $L \leq 150 \mathrm{~mm}$ (see Appendix A). This detail is classified as FAT40 according to EN1993-1-9 [2], as FAT 50 (with CAFL=29 MPa) according to IIW recommendations [10], and as FAT56 (with $\mathrm{CAFL}=31$ $\mathrm{MPa}$ ) according to AASHTO bridge design specifications [12];

2. From CA fatigue testing of 26 end-welded cover plate beams having beam flange thickness, $t_{f}=9.78 \mathrm{~mm}$ and cover-plate thickness, $13.1 \mathrm{~mm} \leq t_{c} \leq 14.3 \mathrm{~mm}$ (see Appendix A). This detail is classified as FAT50 according to EN1993-1-9 [2]. Since the CA experimental dataset considered has not the same classification as the VA experimental data-set (FAT45), the observed stress ranges $S_{i}$ are reduced by a factor $(45 / 50=0.9)$ to account for size effect and then to be consistent with the VA experimental data-set detail classification. Note that the very same detail is classified as FAT 50 (with $\mathrm{CAFL}=29 \mathrm{MPa}$ ) according to IIW recommendations [10], where the influence of $t_{c}$ and $t_{f}$ is not taken into account, and as FAT 40 (with $\mathrm{CAFL}=18 \mathrm{MPa}$ ) according to AASHTO bridge design specifications [12], where the influence of $t_{c}$ and $t_{f}$ is stronger than in Eurocode standards.

These data-sets are reported, with some differences/errors, in the Commentary to Eurocode 3 [2]. Errors above are corrected in Appendix A. The CA characteristic S-N curves were computed for both details by using the MLMCS approach presented in Section 2.1 and compared to the characteristic $\mathrm{S}-\mathrm{N}$ curves from current standards.

The parameters $\Delta m, \mu_{D}, \sigma_{D}$ of the VA stochastic model were estimated by fitting the following VA experimental data-sets:

1. From VA fatigue testing of 21 welded in-plane gussets, under 9 different three-blocks stress range spectra with a block length ranging between 800 and $152 \cdot 10^{3}$ cycles and constant relative damage $\left(S_{i}^{m_{1}} \cdot n_{i} / S_{\max }\right)$ (ICOM tests, [25]). (see Appendix B) The attachment length, $L$, is equal to $150 \mathrm{~mm}$;

2. From VA fatigue testing of 32 end-welded cover plate beams, under 5 different wide-band Rayleigh-type stress range spectra with a block length ranging between 1001 and 10001 cycles [13] (see Appendix B). The thickness of the beam flange and of the cover plate is $25.4 \mathrm{~mm}$. 


\begin{tabular}{rcccc} 
Parameter & \multicolumn{2}{c}{$\begin{array}{c}\text { ML estimate } \\
V=\mathcal{N}\end{array}$} & \multicolumn{2}{c}{$\begin{array}{c}\text { ML estimate } \\
\end{array}$} \\
\cline { 2 - 5 } & MLE & St.Dev. & MLE & St.Dev. \\
\hline$m_{0}$ & 25.770 & 0.945 & 25.804 & 0.954 \\
$m_{1}$ & -2.666 & 0.209 & -2.674 & 0.211 \\
$\sigma$ & -1.048 & 0.144 & -1.048 & 0.144 \\
$\mu_{V}$ & 3.864 & 0.127 & 3.966 & 0.104 \\
$\sigma_{V}$ & -1.667 & 0.498 & -1.712 & 0.590 \\
\hline \hline$\hat{L} L$ & \multicolumn{2}{|c}{12.34} & \multicolumn{2}{c}{13.52}
\end{tabular}

Table 2: In-plane gusset, CA S-N model parameters

Since the estimated CA S-N stochastic models were used for ML-MCS estimation of VA S-N stochastic model, VA details were chosen so that they consistently have the same Eurocodes, IIW and AASHTO FAT classification of the CA details.

VA characteristic S-N curves were computed for both details by using MLMCS approach presented in Section 2.2 and compared to characteristic S-N curves from current standards.

\section{Results}

\section{1. $C A S-N$ curves}

The ML estimate and the standard deviation of the CA S-N model vector parameters are shown in Table 2 for the in-plane gusset data-set and in Table 3 for the cover plate data-set. The correlation coefficients $c_{i j}=\rho_{i j} / \sqrt{\rho_{i i} \rho_{j j}}$ of the CA S-N model are shown in Table 4 for the in-plane gusset data-set and in Table 5 for the cover plate data-set.

ML-MCS-based characteristic CA S-N curves are shown in Figure 7 for the in-plane gusset detail and in Figure 8 for the cover plate detail. For both details the case $V=\mathcal{N}$, having the best ML fit, is presented. Comparison with characteristic S-N curves from standards is also shown. It is remarked that the MCS scheme used for estimation of characteristic S-N curves takes into account both: 1) The epistemic uncertainty of S-N model parameters; and 2) The aleatory uncertainty of the CAFL and of the fatigue life. Nested sampling of the epistemic uncertainty and of the aleatory uncertainty allows for computing true $p$-quantiles of the fatigue life. 


\begin{tabular}{|c|c|c|c|c|}
\hline \multirow[t]{2}{*}{ Parameter } & \multicolumn{2}{|c|}{$\begin{array}{c}\text { ML estimate } \\
\qquad V=\mathcal{N}\end{array}$} & \multicolumn{2}{|c|}{$\begin{array}{c}\text { ML estimate } \\
\qquad V=\mathrm{EV}\end{array}$} \\
\hline & MLE & St.Dev. & MLE & St.Dev. \\
\hline$m_{0}$ & 28.657 & 2.852 & 28.775 & 2.920 \\
\hline$m_{1}$ & -3.416 & 0.786 & -3.448 & 0.804 \\
\hline$\sigma$ & -0.544 & 0.197 & -0.540 & 0.199 \\
\hline$\mu_{V}$ & 3.451 & 0.0577 & 3.540 & 0.0679 \\
\hline$\sigma_{V}$ & -1.623 & 0.408 & -1.592 & 0.440 \\
\hline$n L L$ & & & & \\
\hline
\end{tabular}

Table 3: Cover plate, CA S-N model parameters

$$
\underline{\underline{c}}=\left[\begin{array}{ccccr}
1.00 & -1.00 & 0.00 & -0.06 & 0.06 \\
\vdots & 1.00 & -0.00 & 0.05 & -0.06 \\
\vdots & \vdots & 1.00 & -0.00 & 0.00 \\
\vdots & \vdots & \vdots & 1.00 & -0.62 \\
\vdots & \vdots & \vdots & \vdots & 1.00
\end{array}\right]
$$

Table 4: In-plane gusset, CA S-N model correlation matrix, $(V=\mathcal{N})$ case

$$
\underline{\underline{c}}=\left[\begin{array}{ccccr}
1.00 & -1.00 & 0.07 & -0.05 & 0.06 \\
\vdots & 1.00 & -0.07 & 0.05 & -0.06 \\
\vdots & \vdots & 1.00 & -0.04 & 0.04 \\
\vdots & \vdots & \vdots & 1.00 & -0.03 \\
\vdots & \vdots & \vdots & \vdots & 1.00
\end{array}\right]
$$

Table 5: Cover plate, CA S-N model corr. matrix, $(V=\mathcal{N})$ case 

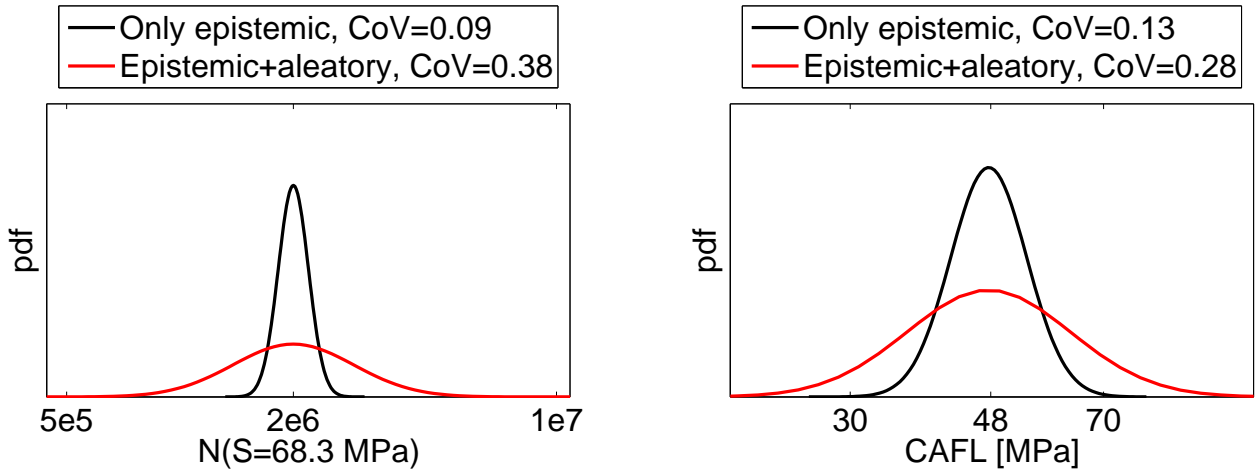

Figure 5: Influence of the aleatory uncertainty of the fatigue life and of the CAFL, in-plane gusset

In order to assess the influence of the aleatory uncertainty following analysis was carried out, by considering the cover-plate and the in-plane gusset datasets:

1. $10^{6} \mathrm{~S}-\mathrm{N}$ curves were sampled by only considering the epistemic uncertainty of S-N model parameters; two vectors of size $\left(10^{6} \times 1\right)$ were created by taking from each sampled curve the CAFL and the number of cycle $N^{*}$ corresponding to the fatigue strength at $2 \cdot 10^{6}$ cycles according to the median $\mathrm{S}-\mathrm{N}$ curve $(S=68.3 \mathrm{MPa}$ for the in-plane gusset, while $S=69.9 \mathrm{MPa}$ for the cover-plate). These vectors were fitted with two log-Normal distributions (CAFL and $N^{*}$ log-Normal distributions);

2. Step 1 was repeated by considering both the epistemic uncertainty of S-N model parameters and the aleatory uncertainty of the fatigue life and of the CAFL;

3. Log-Normal distributions of the CAFL and of $N^{*}$ estimated at Step 1 were compared to log-Normal distributions of the CAFL and of $N^{*}$ estimated at Step 2, in terms of the coefficient of variation (see Figures 5 and 6$)$.

\subsection{VA $S-N$ curves}

VA S-N models were estimated for the two considered details, using the ML-MCS scheme presented in Section 2.2.

A sensitivity study was carried out for choosing the sampling parameters 

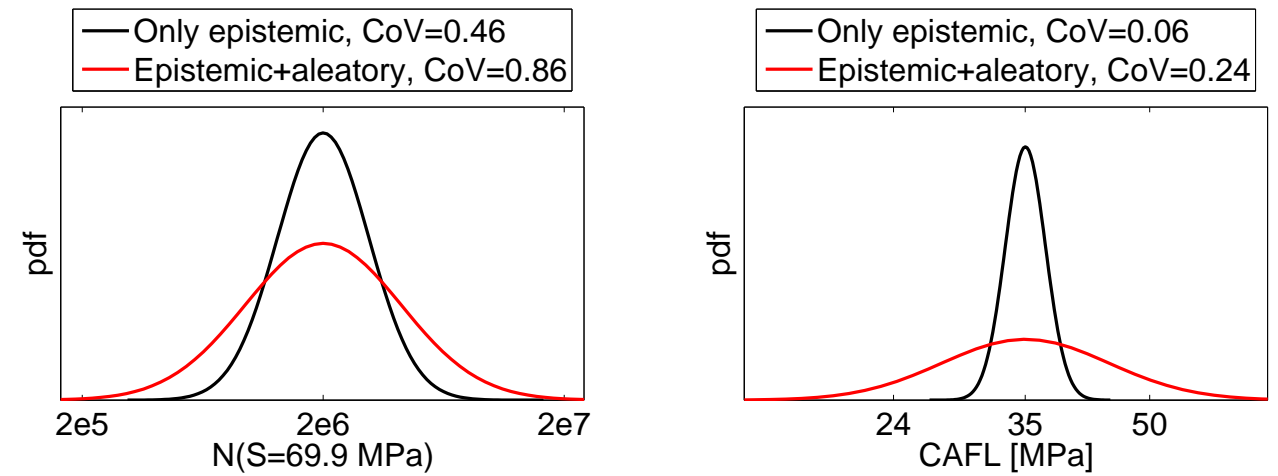

Figure 6: Influence of the aleatory uncertainty of the fatigue life and of the CAFL, coverplate

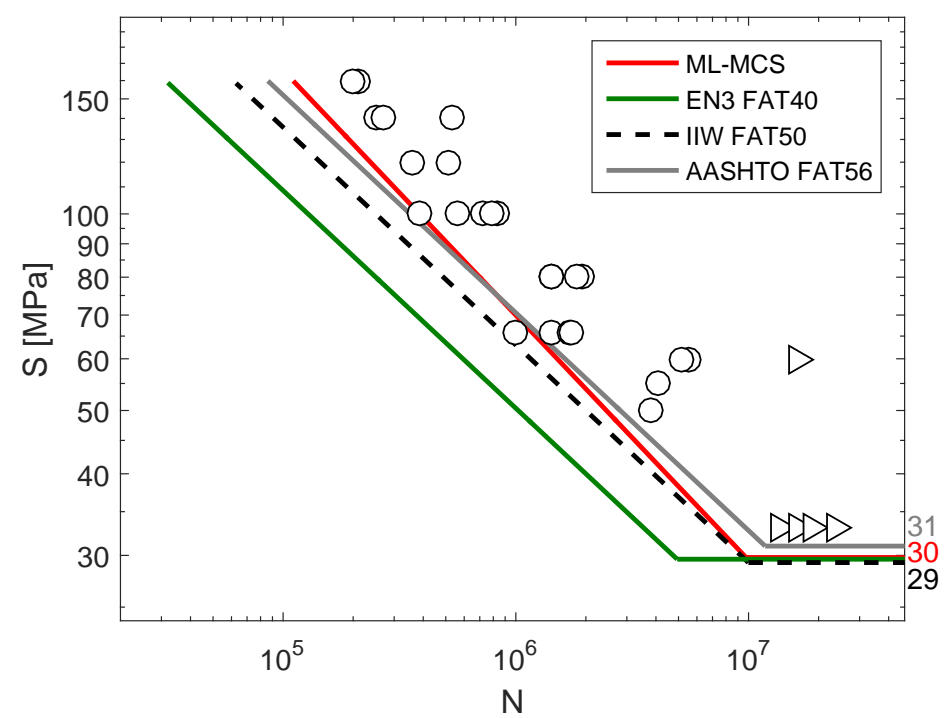

Figure 7: In-plane gusset, characteristic S-N curves 


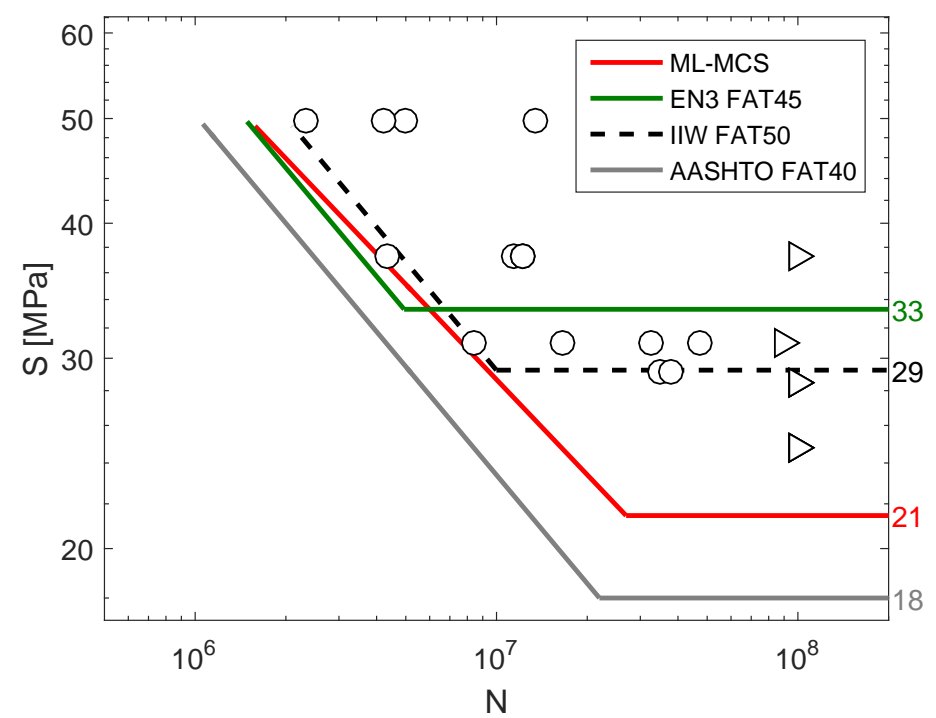

Figure 8: Cover plate, characteristic S-N curves

$\eta_{\text {iter }}=5000, \eta_{\text {res }}=100$ to be used for the estimation of VA S-N models.

Three cut-off cases (No cut-off, $\mathrm{S}_{\mathrm{co}}=14.6 \mathrm{MPa}, \mathrm{S}_{\mathrm{co}}=0.5 \cdot \mathrm{CAFL}$ ) and two critical damage sum distributions $\left(D_{c}=\log \mathcal{N}, D_{c}=\mathcal{W}\right)$ were considered. The combination $\eta_{\text {iter }}=5000, \eta_{\text {res }}=100$, which gives a variation in $\overline{n \hat{L} L}$ lower than $0.2 \%$, was chosen for the estimation of VA S-N models.

The ML estimate of the slope range, $\Delta \hat{m}$, was estimated by producing notched box plots at the several values of $\Delta m$ and by choosing the lowest $\overline{\hat{n} L L}(\Delta m)$ with $5 \%$ significance level (see Figures 9 and 10).

The ML estimates of parameters $\mu_{D}, \sigma_{D}, \Delta m$ and the value of the negative log-Likelihood at ML estimates, for the in-plane gusset data-set, are shown in Table 6. The combination (no cut-off $/ D_{c}=\log \mathcal{N}$ ) gives the best fit in terms of negative log-Likelihood. Between the two cut-off cases, the case $S_{c o}=14.6$ $\mathrm{MPa}$ gives the best fit in terms of negative log-Likelihood. Correlation coefficients between model parameters are shown in Table 7. Figure 11 shows the ML-MCS-based VA characteristic S-N curve for the in-plane gusset detail. The combination no cut-off / $D_{c}=\log \mathcal{N}$, which gave the best ML fit of VA test results, was chosen. The slope $m_{2}$ is equal to -7.7 and the median value of the critical damage sum, $d_{c, 5}$, is equal to 0.74. The ML-MCS-based VA S-N curve was re-scaled according to Equation 12 for direct comparison with 


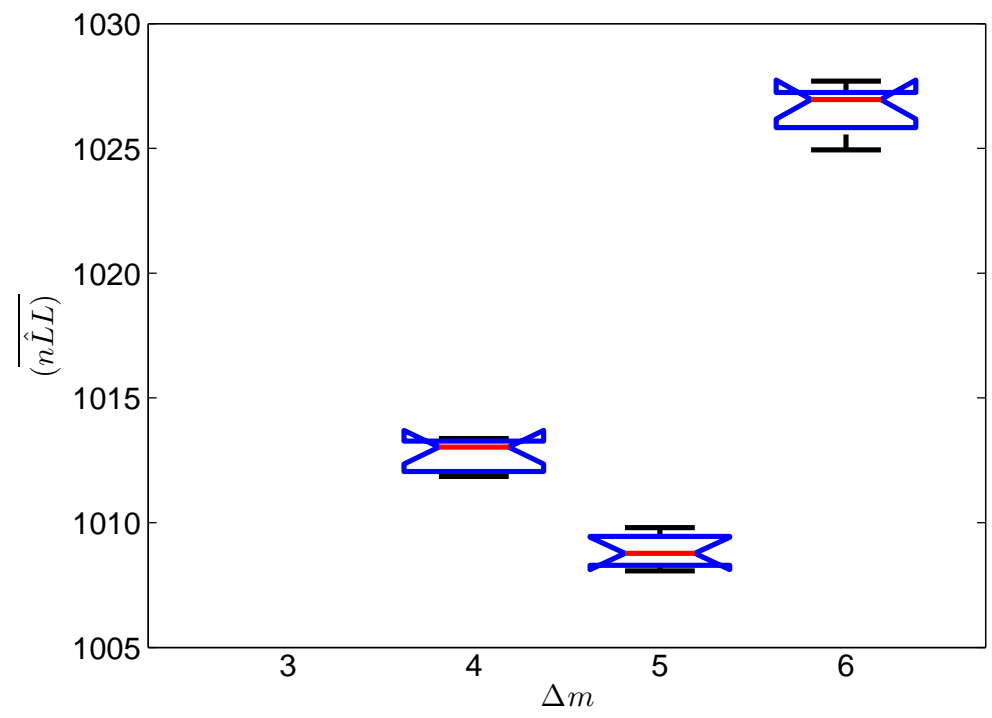

Figure 9: In-plane gusset, negative log-Likelihood notched box plot, no cut-off $/ D_{c}=$ $\log \mathcal{N}$

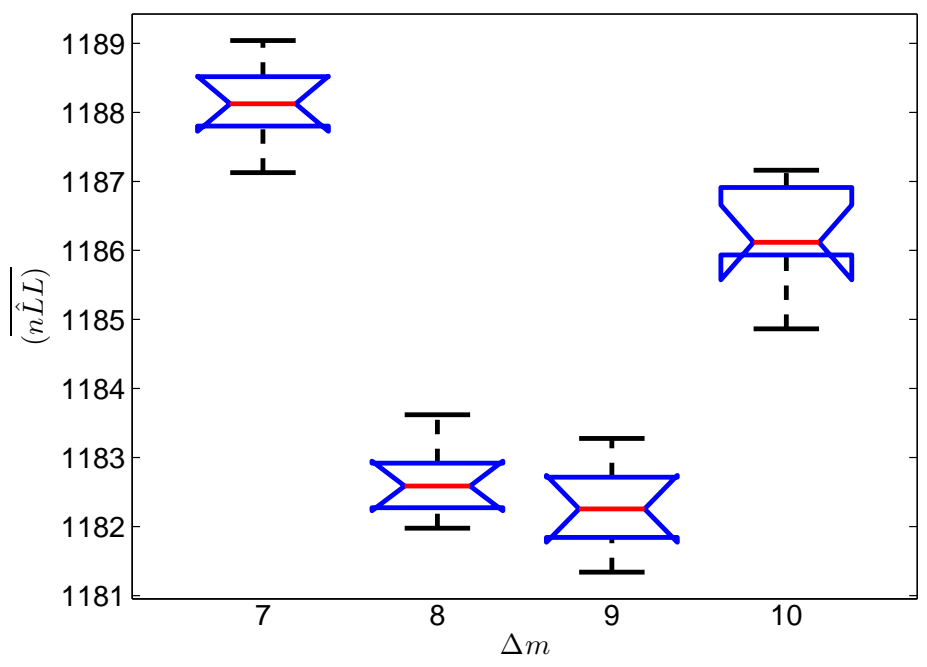

Figure 10: Cover plate, negative log-Likelihood notched box plot, no cut-off $/ D_{c}=\mathcal{W}$ 


\begin{tabular}{|c|c|c|c|c|c|c|c|c|c|c|}
\hline Cut-off case & $D_{c}$ & $\hat{m}_{o}$ & $\hat{m}_{1}$ & $\hat{\sigma}$ & $\hat{\mu}_{V}$ & $\hat{\sigma}_{V}$ & $\hat{\mu}_{D}$ & $\hat{\sigma}_{D}$ & $\Delta \hat{m}$ & $\overline{n \hat{L} L}$ \\
\hline & $\log \mathcal{N}$ & \multirow{6}{*}{25.77} & \multirow{6}{*}{-2.67} & \multirow{6}{*}{-1.05} & \multirow{6}{*}{3.86} & \multirow{6}{*}{-1.67} & -0.31 & 0.60 & 5 & 1009 \\
\hline no cut-oft & $\mathcal{W}$ & & & & & & 1.00 & 1.48 & 5 & 1343 \\
\hline $146 \mathrm{MPa}$ & $\log \mathcal{N}$ & & & & & & -0.30 & 0.60 & 5 & 1009 \\
\hline $14.0 \mathrm{MIPa}$ & $\mathcal{W}$ & & & & & & 1.00 & 1.48 & 5 & 1343 \\
\hline \multirow{2}{*}{$0.5 \cdot \mathrm{CAFL}$} & $\log \mathcal{N}$ & & & & & & -0.26 & 0.61 & 5 & 1117 \\
\hline & $\mathcal{W}$ & & & & & & 1.07 & 1.40 & 5 & 1486 \\
\hline
\end{tabular}

Table 6: In-plane gusset, VA S-N curve parameters

$$
\underline{\underline{c}}=\left[\begin{array}{ccccccc}
1.00 & -1.00 & 0.00 & -0.05 & 0.06 & -0.01 & 0.01 \\
\vdots & 1.00 & -0.00 & 0.05 & -0.06 & 0.00 & 0.00 \\
\vdots & \vdots & 1.00 & -0.01 & 0.01 & 0.01 & 0.02 \\
\vdots & \vdots & \vdots & 1.00 & -0.62 & -0.08 & -0.02 \\
\vdots & \vdots & \vdots & \vdots & 1.00 & 0.04 & 0.02 \\
\vdots & \vdots & \vdots & \vdots & \vdots & 1.00 & 0.37 \\
\vdots & \vdots & \vdots & \vdots & \vdots & \vdots & 1.00
\end{array}\right]
$$

Table 7: In-plane gusset, VA S-N model correlation matrix, no cut-off $/ D_{c}=\log \mathcal{N}$ 


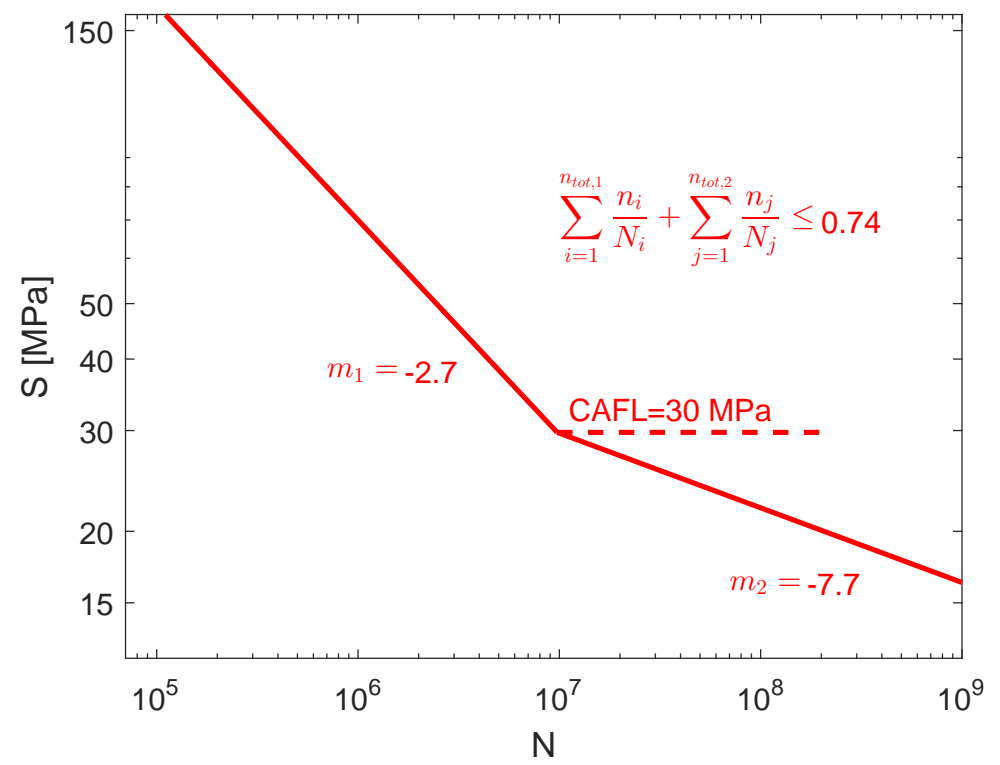

Figure 11: In-plane gusset, MCS-ML characteristic VA S-N curve, no cut-off / $D_{c}=\log \mathcal{N}$

standard VA S-N curves (see Figure 12). The ML estimates of parameters $\mu_{D}, \sigma_{D}, \Delta m$ and the value of the negative log-Likelihood at ML estimates, for the cover-plate data-set, are shown in Table 8. The combination no cut-off $/ D_{c}=\mathcal{W}$ gives the best fit in terms of negative log-Likelihood. Between the two cut-off cases, the case $S_{c o}=14.6 \mathrm{MPa}$ gives the best fit in terms of negative log-Likelihood. Correlation coefficients between model parameters are shown in Table 9. The assumption of asymptotic normality of the multivariate vector $\left(\hat{\mu}_{D}, \hat{\sigma}_{D}, n \hat{L} L\right)$ was verified for both the cover-plate data-set and the in-plane gusset data-set, by using density and probability plots.

When observing ML-MCS-based characteristic failure damage sums for the cover-plate data-set (see first column of Table 10), it is evident that the lowest damage sum (third spectrum in B.15, failure at $104 \cdot 10^{6}$ cycles) lies an abnormal distance from other damage sum values. In order to assess whether this value can be classified as an outlier, the box-plot method was applied to observed failure log-damage sums. Results of the analysis are shown in Table 10: since the lowest failure log-damage sum (-2.60) exceeds the lower outer fence $\left(F_{l o}\right)$, it can be classified as extreme outlier. The cover-plate VA data-set was re-analysed by neglecting the failure point corresponding 


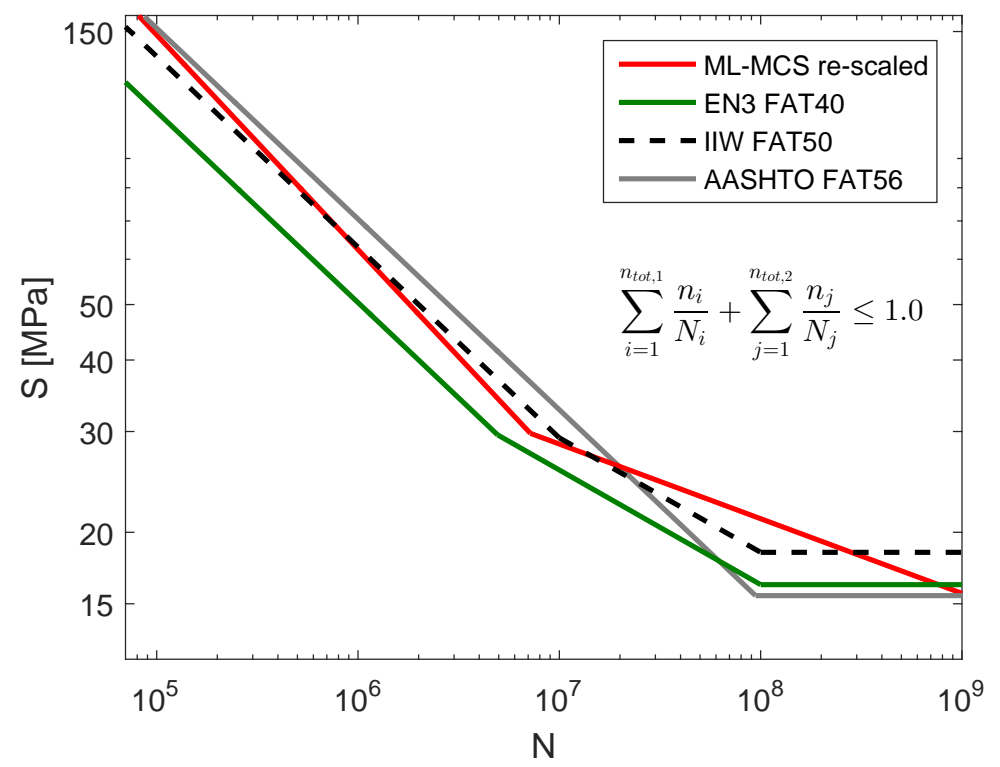

Figure 12: In-plane gusset, characteristic VA S-N curves

\begin{tabular}{|c|c|c|c|c|c|c|c|c|c|c|}
\hline Cut-off case & $D_{c}$ & $\hat{m}_{o}$ & $\hat{m}_{1}$ & $\hat{\sigma}$ & $\hat{\mu}_{V}$ & $\hat{\sigma}_{V}$ & $\hat{\mu}_{D}$ & $\hat{\sigma}_{D}$ & $\Delta \hat{m}$ & $\overline{n \hat{L} L}$ \\
\hline & $\log \mathcal{N}$ & \multirow{6}{*}{28.65} & \multirow{6}{*}{-3.42} & \multirow{6}{*}{-0.54} & \multirow{6}{*}{3.45} & \multirow{6}{*}{-1.62} & 0.50 & 1.46 & 7 & 1431 \\
\hline no cu & $\mathcal{W}$ & & & & & & 1.70 & 1.40 & 8 & 1183 \\
\hline \multirow{2}{*}{$14.6 \mathrm{MPa}$} & \multirow{2}{*}{$\begin{array}{c}\log \mathcal{N} \\
\mathcal{W}\end{array}$} & & & & & & 0.50 & 0.87 & 4 & 1339 \\
\hline & & & & & & & 1.75 & 1.61 & 7 & 1190 \\
\hline \multirow{2}{*}{$0.5 \cdot \mathrm{CAFL}$} & $\log \mathcal{N}$ & & & & & & 0.59 & 1.24 & 6 & 1482 \\
\hline & $\mathcal{W}$ & & & & & & 2.13 & 1.28 & 8 & 1324 \\
\hline
\end{tabular}

Table 8: Cover plate, VA S-N curve parameters 


$$
\underline{\underline{c}}=\left[\begin{array}{ccccccr}
1.00 & -1.00 & 0.08 & -0.05 & 0.06 & -0.03 & 0.01 \\
\vdots & 1.00 & -0.07 & 0.05 & -0.06 & 0.02 & 0.01 \\
\vdots & \vdots & 1.00 & -0.04 & 0.04 & 0.02 & 0.00 \\
\vdots & \vdots & \vdots & 1.00 & -0.03 & -0.00 & -0.01 \\
\vdots & \vdots & \vdots & \vdots & 1.00 & 0.03 & -0.00 \\
\vdots & \vdots & \vdots & \vdots & \vdots & 1.00 & -0.71 \\
\vdots & \vdots & \vdots & \vdots & \vdots & \vdots & 1.00
\end{array}\right]
$$

Table 9: Cover plate, VA S-N model correlation matrix, no cut-off $/ D_{c}=\mathcal{W}$

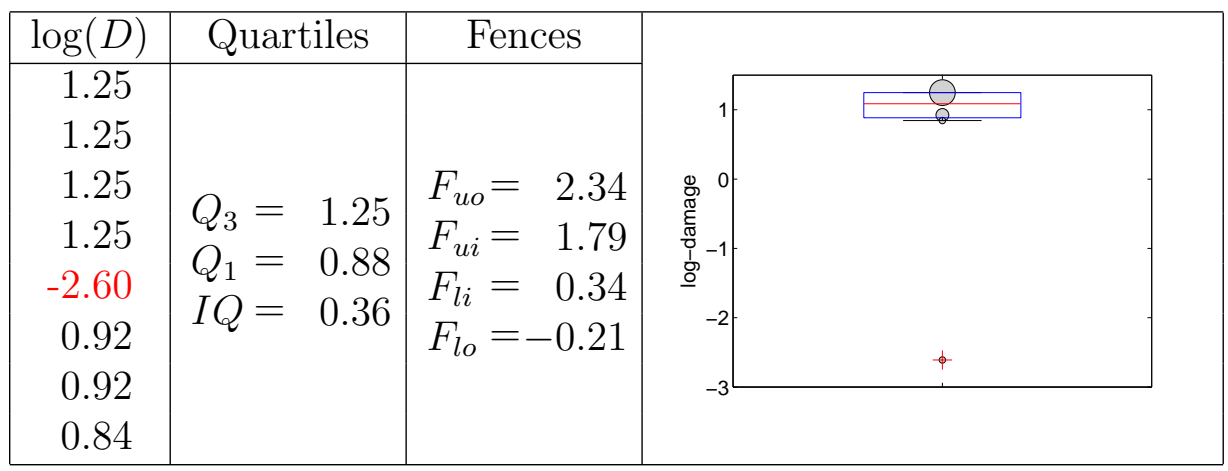

Table 10: Cover plate, outlier disqualification

to the classified outlier: the ML estimates of parameters of $D_{c}$ distribution and the ML estimate of slope change, $\hat{\Delta} m$ are shown in Table 11. Figure 13 shows the ML-MCS-based VA characteristic S-N curve for the cover-plate detail. The combination no cut-off / $D_{c}=\log \mathcal{N}$, which gave the best ML fit of VA test results after outlier disqualification, was chosen. The slope $m_{2}$ is equal to -9.4 and the median value of the critical damage sum, $d_{c, .5}$, is equal to 1.31. The ML-MCS-based VA S-N curve was re-scaled according to Equation 12 for direct comparison with standard VA S-N curves (see Figure

\begin{tabular}{c|cc|rrrr} 
Outlier disqualification & Cut-off case & $D_{c}$ & $\hat{\mu}_{D}$ & $\hat{\sigma}_{D}$ & $\Delta \hat{m}$ & $\overline{\hat{n} L L}$ \\
\hline no & no cut-off & $\mathcal{W}$ & 1.70 & 1.40 & 8 & 1183 \\
\hline yes & no cut-off & $\log \mathcal{N}$ & 0.27 & 0.58 & 6 & 934 \\
\hline
\end{tabular}

Table 11: Cover-plate, outlier disqualification: models comparison 


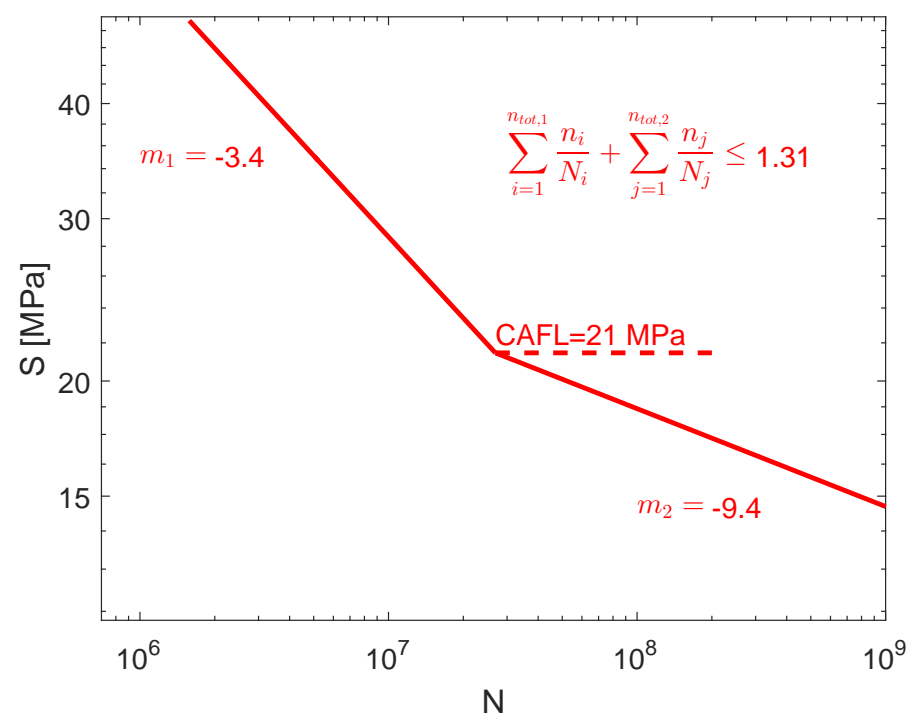

Figure 13: Cover plate, MCS-ML characteristic VA S-N curve, no cut-off $/ D_{c}=\log \mathcal{N}$

14). Figure 15 shows the ML-MCS-based pdfs of $D_{c}$, for the in-plane gusset detail and the cover-plate detail; comparison with conventional $\log \mathcal{N}(0,0.3)$ damage distribution (red shaded pdf) is also provided.

\section{Discussion}

In this paper a new probabilistic approach for estimation of CA and VA $\mathrm{S}-\mathrm{N}$ curves of welded components was presented, with application to two study cases.

Concerning CA S-N curves, results presented in Section 4.1 provide following indications:

- For both details, modeling the CAFL as $V=\mathcal{N}$ in ML-MCS based $\mathrm{S}-\mathrm{N}$ curves, gives a better fit in terms of sample log-Likelihood, with respect to the case $(V=\mathrm{EV})$ (see Tables 2 and 3 );

- For both details, the intercept, $m_{0}$, and the slope, $m_{1}$, of the ML-MCS based S-N curves are fully linearly correlated. Also, for both details there is no linear correlation between: 1) The S-N curve variance parameter, $\sigma$, and all other parameters; and 2) The S-N curve parameters 


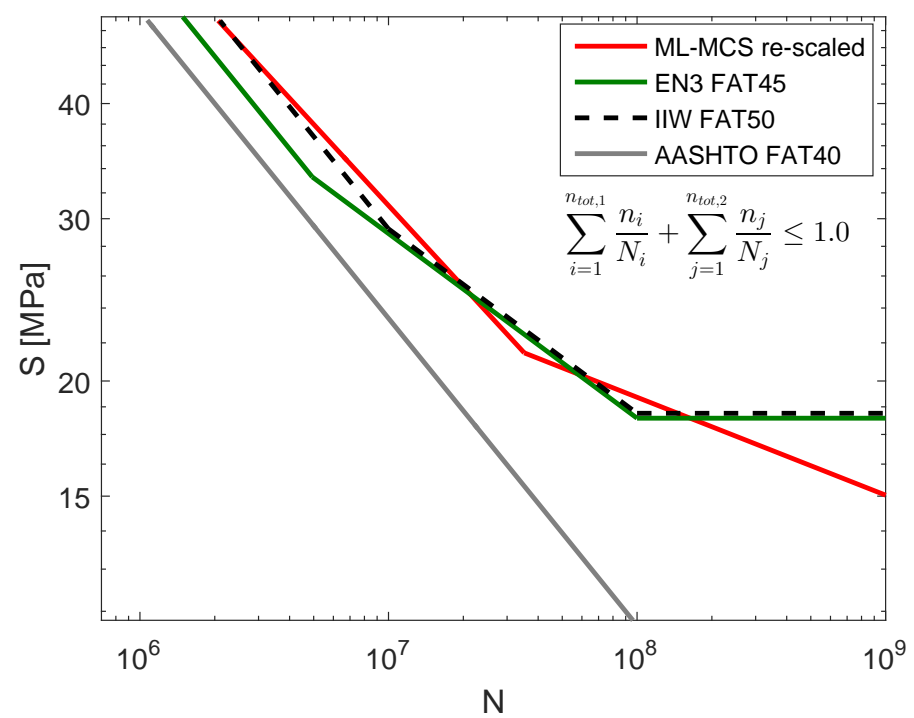

Figure 14: Cover plate, characteristic VA S-N curves, no cut-off $/ D_{c}=\log \mathcal{N}$

$m_{0}, m_{1}$ and the log-CAFL parameters $\mu_{V}, \sigma_{V}$. Furthermore, the coverplate detail shows no linear correlation between the location parameter and the scale parameter of the log-CAFL probability distribution while the in-plane gusset data-set shows moderate negative linear dependency between these two parameters (see Tables 4 and 5);

- The study of the epistemic and aleatory randomness shows the strong influence of the aleatory randomness of the fatigue life and of the CAFL, respectively on the $N$ distribution (the coefficient of variation increases from 0.09 to 0.38 for the in-plane gusset and from 0.46 to 0.86 for the cover-plate, see Figures 5 and 6 ) and on the CAFL distribution (the coefficient of variation increases from 0.13 to 0.28 for the in-plane gusset and from 0.06 to 0.24 for the cover-plate, see Figures 5 and 6)

- The ML-MCS characteristic S-N curves for two considered details were linearized for direct comparison with current standards. Eurocode 1993-1-9 standards, AASHTO bridge design specifications and IIW recommendations provide estimation of fatigue strength in the FL region $\left(\mathrm{N}<5 \cdot 10^{6}\right.$ cycles) which is similar to the estimation based on ML-MCS approach (see Figures 7 and 8). 
In-plane gusset, $d_{c, .05}=0.78$

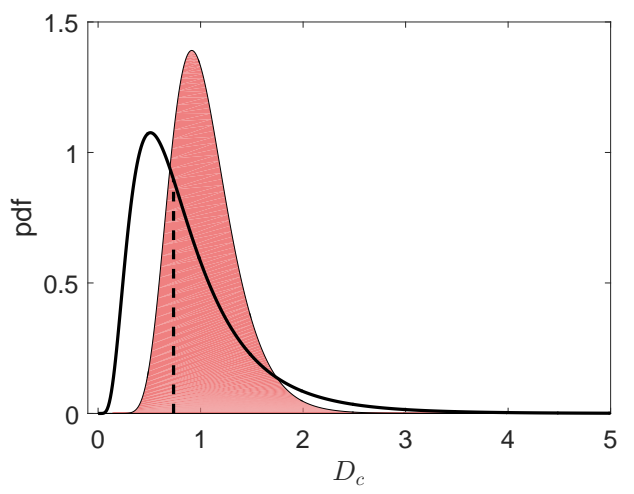

Cover plate, $d_{c, 05}=1.31$

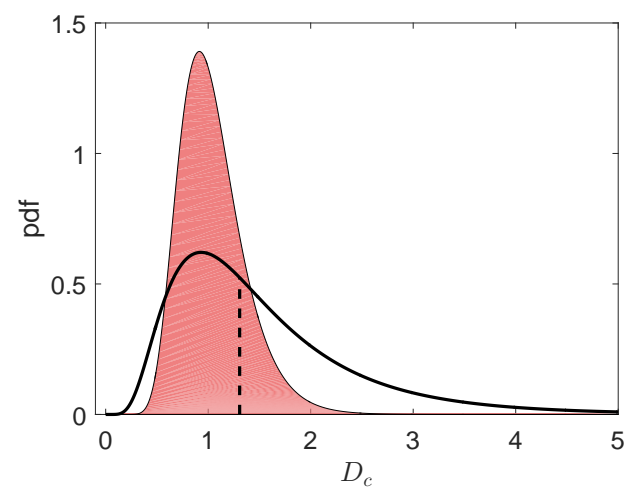

Figure 15: ML-MCS based pdfs of $D_{c}$. Shaded red is the pdf of $\log \mathcal{N}(0,0.3)$ distrib.

A larger difference can be observed in the $\mathrm{HCF}$ region: for the cover plate detail both Eurocode and AASHTO-based S-N curves provide unsafe estimation of the CAFL and of the knee point (with significant amount of experimental failure points lying below the CAFL), while the AASHTO-based S-N curve provides over-conservative estimation of the CAFL (see Figure 8); for the in-plane gussets all standards provide very similar estimation of CAFL (see Figure 7);

- The assumption of having the knee point at a number of cycles which increases as the fatigue strength at 2 million cycles decreases (as assumed in AASHTO specifications) seems to be confirmed by estimated ML-MCS S-N curves (in-plane gusset has FAT $=54$ and knee point at $9.8 \cdot 10^{6}$ cycles, while the cover plate has $\mathrm{FAT}=46$ and knee point at $2.7 \cdot 10^{7}$ cycles). This has to be confirmed by further experimental results.

Concerning VA S-N curves, results presented in Section 4.2 provide following indications:

- The estimates of VA S-N stochastic model for both in-plane gusset and cover-plate data-sets (see Tables 6 and 8) show that the VA S-N curve without stress range cut-off maximizes the Likelihood information: the modeling of a cut-off at $14.6 \mathrm{MPa}$ (lowest Eurocode FAT detail) and a cut-off at $0.5 \cdot \mathrm{CAFL}$, in conjunction with $m_{2}<<-3$, are not supported by VA experimental results. Also, this indicates that experimental try- 
outs with lower cut-off values should be tried. Indeed, Baptista [25] found experimentally on in-plane gusset detail a cut-off limit;

- For the in-plane gusset data-set, the graph $\overline{n \hat{L} L}-\Delta m$ shows that $D_{c}=\log \mathcal{N}$ with $\Delta \hat{m}=5$ gives the best fit in terms of Likelihood (see Figure 9. For the cover plate data-set, the analysis of experimental points by using box-plot method allowed to classify one failure points as a extreme outlier: after outlier disqualification, the graph $\overline{n \hat{L} L}-\Delta m$ shows that $D_{c}=\log \mathcal{N}$ with $\Delta \hat{m}=6$ gives the best fit in terms of Likelihood (see Table 11). The disqualification of the outlier point does not affect the median value of $D_{c}$ distribution (which remains equal to 1.31). These values are higher then Haibach proposition (recommended in the Eurocode 3 Part 1-9 [9]) of $\Delta m=2$;

- ML fit provides support $D_{c}=\log \mathcal{N}$ for both the in-plane gusset and for the cover-plate data-sets, once disqualification of one extreme outlier of the cover-plate data-set is made;

- The VA S-N model correlation matrix shows that there is no linear correlation between the parameters of the S-N curve and the parameters of the $D_{c}$ distribution, for both in-plane gusset and cover plate datasets (see Tables 7 and 9). This seems to indicate that the location and the scale parameter of $D_{c}$ are influenced only by the characteristics of loading history and not by the fatigue strength of the studied detail. Further analysis of different fatigue details is needed to confirm this indication;

- For the cover plate data-set, where experimental results were obtained under wide-band Rayleigh type stress range spectra having short block lengths, the median value of $D_{c}$ is equal to 1.31 , while for the in-plane gusset study case, where experimental results were obtained under 3blocks spectra with stress ranges cycling down from constant maximum stress (no over-loads which could retardate the crack opening), the median value of $D_{c}$ is equal to 0.74 . This seems to indicate that there is a strong influence of loading characteristics and in particular of load sequence on the critical damage sum distribution, which is coherent with results from [16] and [15];

- The slope range $\Delta m=m_{1}-m_{2}$ is equal to 5 for the in-plane gusset, 
while it is equal to 6 for the cover-plate: this confirms the existence of a second slope $m_{2}$ (in disagreement with results from [13], [14], [16] and [17]) and it seems to indicate that the influence of the loading characteristics on the second slope $m_{2}$ is small;

- Re-scaling of ML-MCS characteristic VA S-N curve at $D_{c}=1$ allowed for direct comparison of ML-MCS-based VA S-N curves with standard VA S-N curves, showing that S-N curve from AASHTO specification is highly over-conservative for the cover plate data-set at all stress ranges (see Figure 14), and that all standard S-N curves (Eurocode, IIW, AASHTO) are slightly over-conservative at stress ranges lower than 30 $\mathrm{MPa}$, for the in-plane gusset data-set (see Figure 12);

\section{Conclusions}

In this paper a new ML-MCS approach was proposed for estimation of: 1) CA S-N curve of welded joints; 2) VA S-N model of welded joints, including both the VA S-N curve and the critical value of damage sum, which are used in Miner's equation when the fatigue life of a welded joint under the effect of VA loading is computed.

The novel contribution of the ML-MCS approach consists in: 1) Using MCS to find true $p$-quantiles of CA S-N curves; 2) Re-simulating VA experimental fatigue tests and in estimating the VA S-N curve second slope, $m_{2}$, and the critical damage sum, $D_{c}$, with ML method. Linearization method is also proposed for direct comparison of ML-MCS characteristic S-N curves with current standards.

The ML-MCS approach constitutes a powerful tool for re-definition of standard CA S-N curves of welded joints, for both new structures as well as existing structures. However, since for new structures the simplicity is prioritized over the accuracy (see Section 1.2), the estimation of more accurate $\mathrm{S}-\mathrm{N}$ curves is more important for existing structures, where reliable assessment of remaining fatigue life is of primary importance. Use of ML method allows to use run-out data for estimation of S-N curves. The novel idea of using MCS technique to estimate true $p$-quantiles of fatigue life gives more insight into realistic estimation of CAFL and knee point position.

According to considered study cases, arbitrarily fixing the CAFL at fixed number of cycles lead to an un-safe assumption which could be extremely dangerous for fatigue verifications based on CAFL. 
It is recalled that the effectiveness of the proposed ML-MCS approach is conditioned to the use of experimental CA data-sets which have significant information in the $\mathrm{HCF}$ region.

The ML-MCS approach constitutes a powerful tool for re-defining the second slope of S-N curves, $m_{2}$, as well as the critical value of the damage sum used in Miner's rule, $D_{c}$. The novel idea of re-simulating experimental VA fatigue tests and to estimate $m_{2}$ and $D_{c}$ with ML method allows one to: 1) Give experimental support to the amount of the reduction of S-N curve slope, $\Delta m=m_{1}-m_{2} ; 2$ ) Give experimental support to the choice of probability distribution of $D_{c}$.

The considered study cases show that the ML-MCS approach gives the most realistic estimation of failure damage sum with respect to existing standards. It is also noted that there is strong influence of load sequence on $D_{c}$ probability distribution; it follows that realistic load histories should be used for producing VA experimental results, when the proposed ML-MCS approach is used for re-definition of $m_{2}$ and $D_{c}$ in current standards.

\section{Future works}

The results of this paper provide a strong foundation for future work in fatigue verification of welded connections, in particular for existing structures. Results have shown that there is a need of more CA and VA fatigue experimental results in the HCF region. Three areas of future work have been identified as follows: 1) Re-definition of CA S-N curves in Eurocode standards for existing structures; 2) Re-definition of Miner's rule in Eurocode standards; and 3) Inclusion of the bi-linear CA S-N model without CAFL into the ML-MCS approach.

In order to re-define CA S-N curves of welded joints in Eurocode standards, a fatigue test program should be carried out according to following steps: 1) Choice of details to include in the re-definition of Eurocode standards (i.e. longitudinal attachment, transversal attachment, cover plate, Tjoint, ... ); 2) CA fatigue testing of selected details. It is recommended to use stress ranges varying from $1.5 \cdot \mathrm{FAT}_{\mathrm{EN}}$ to $0.5 \cdot \mathrm{CAFL}_{\mathrm{EN}}$, where $\mathrm{FAT}_{\mathrm{EN}}$ and $\mathrm{CAFL}_{\mathrm{EN}}$ are respectively the FAT and the CAFL of tested details, according to EN 1993-1-9. A minimum number of three data points is required for each stress range level. Experimental tests in which $N=10^{8}$ will be reached without failure will be classified as run-outs. Use of the scheme recommended above will ensure significant CA experimental results in the HCF region; 3) 
Estimation of CA S-N stochastic models as well as characteristic S-N curves of considered details, using ML-MCS approach.

Once the CA S-N stochastic model has been estimated for the selected details, a VA fatigue test program needs to be carried out in order to redefine $\mathrm{S}-\mathrm{N}$ curve second slope, $m_{2}$, and critical damage, $D_{c}$. The following steps should be followed:

1. VA fatigue testing of selected details. Due to the strong influence of load history on the critical damage sum $D_{c}$, it is recommended to use realistic loading spectra for VA tests; one possibility would be to repeatedly apply real road bridge recorded load histories to to the tested specimens.

It is recommended to use a number of cycles, $\tilde{n}_{t o t}$, for each loading spectrum, such that:

$$
\int_{\exp \left(\hat{\mu}_{V}\right)}^{s_{\max }} \frac{\tilde{n}_{t o t} \cdot \tilde{f}_{S}(s)}{N(s)} \mathrm{d} s \leq 3.0
$$

where $\widetilde{f}_{S}(s)$ is the loading spectrum, $s_{\max }$ is the maximum stress range of the loading spectrum, $\hat{\mu}_{V}$ is the ML estimate of median value of $\log$-CAFL and $N(s)$ is the number of cycles to failure according to median CA S-N curve ${ }^{7}$. Experimental tests in which $n_{\text {tot }}$ will be reached without failure will be classified as run-outs. Use of the scheme recommended above will ensure to have significant VA experimental results in the $\mathrm{HCF}$ region.

It is recommended to use stress range loading spectra which have $\varsigma$ varying from 0.5 to 0.05 , where $\varsigma$ is the percentage of stress range cycles exceeding the characteristic value of the ML-MCS based CAFL. A minimum number of five different load spectra should be used and a minimum number of three data points is required for each stress range loading spectrum;

\footnotetext{
${ }^{7}$ According to results presented in Section $4, d_{c}=3.0$ is higher than the 0.9 quantile of $D_{c}$ distribution for both cover-plate and in-plane gusset study cases: the choice of $d_{c}=3.0$ in Equation 13 will then ensure to have a significant run-out if the experimental test ends without a failure after $\tilde{n}_{t o t}$ cycles
} 


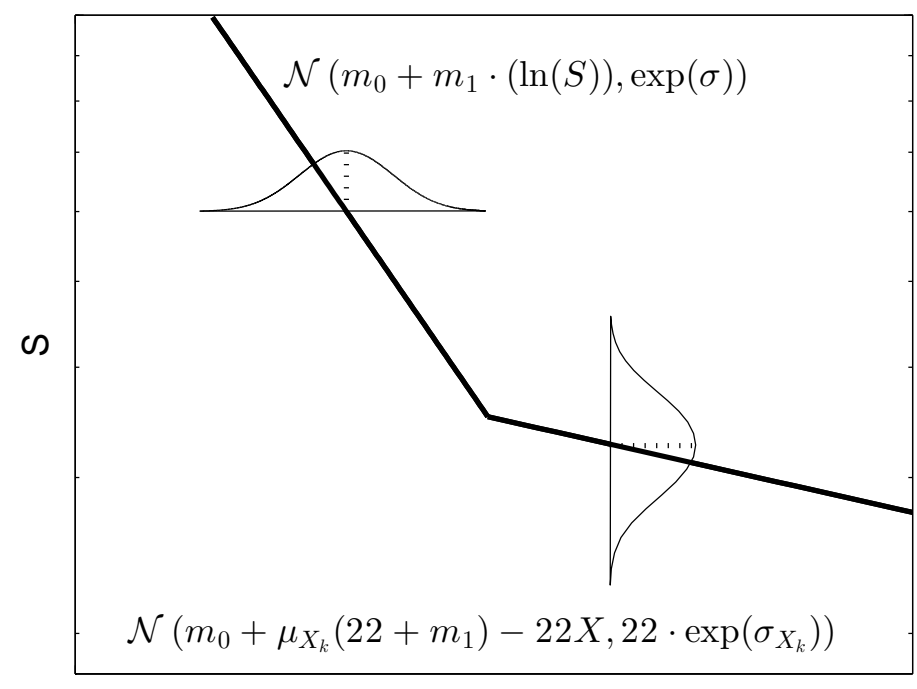

$\mathrm{N}$

Figure 16: Bi-linear S-N curve without CAFL

2. Estimation of VA S-N stochastic models of considered details, which include $m_{2}=\left(m_{1}-\Delta m\right)$ and $D_{c}$, using ML-MCS approach.

The ML-MCS approach presented in this paper is based on linear CA S-N curve having random CAFL. The existence of CAFL is still a topic for debate; Sonsino [8] suggests that CAFL does not exist and that stress range cycles below the knee point should be accounted with a slope $m_{1}^{\prime}=-22$. The knee point is arbitrarily fixed at $10^{7}$ cycles; due to the lack of experimental results in the $\mathrm{HCF}$ region, $m_{1}^{\prime}=-22$ is arbitrarily chosen in order to have a $10 \%$ constant stress range decrease with respect to the $\log (N)$ axis. The ML-MCS approach can be adapted to a bi-linear S-N model having a slope $m_{1}^{\prime}=-22$ for stress range cycles below the knee point. The bi-linear model should be defined as follows:

$$
Y= \begin{cases}m_{0}+m_{1} X+\epsilon(0, \exp (\sigma)), & \text { for } X>X_{k} \\ m_{0}+\mu_{X_{k}}\left(22+m_{1}\right)-22 X+\epsilon\left(0,22 \cdot \exp \left(\sigma_{X_{k}}\right)\right), & \text { for } X \leq X_{k}\end{cases}
$$

where $\mu_{K}$ and $\exp \left(\sigma_{K}\right)$ are respectively the location and the scale parameters of the $X_{k}$ random variable, which represents the natural logarithm of the stress range at the knee point (see Figure 16). 
Thereafter, the model parameter vector, $\underline{\theta}$, relative to the $\mathrm{S}-\mathrm{N}$ model with random CAFL, should be transformed as follows:

$$
\underline{\theta}=\left(m_{0}, m_{1}, \sigma, \mu_{V}, \sigma_{V}\right) \quad \rightarrow \quad \underline{\theta}=\left(m_{0}, m_{1}, \sigma, \mu_{X_{k}}, \sigma_{X_{k}}\right)
$$

As for the case of the S-N model with CAFL, the ML-MCS approach has the advantage of estimating the knee point instead of arbitrarily assuming its position.

Future work is required for: 1) Implementing the CA bi-linear S-N model into the ML-MCS approach; and 2) Validating it with experimental datasets which contain significant information in the HCF region.

\section{Acknowledgements}

This research was supported by the Swiss Federal Roads Office (FEDRO Project AGB 2010/003). The authors would also like to thank Professor M. H. Faber for fruitful discussions and for his valuable comments which contributed to the development of the ML-MCS approach.

\section{Appendix A. CA experimental data-sets}

In this section the two analysed CA experimental data-sets are presented. The applied stress range, $S$, the number of cycles to the end of the test, $N$, the test outcome, $\delta$ and the failure location are presented in Tables A.12 and A.13. The two considered details are shown in Figures A.17 and A.18 with relevant geometrical features. 


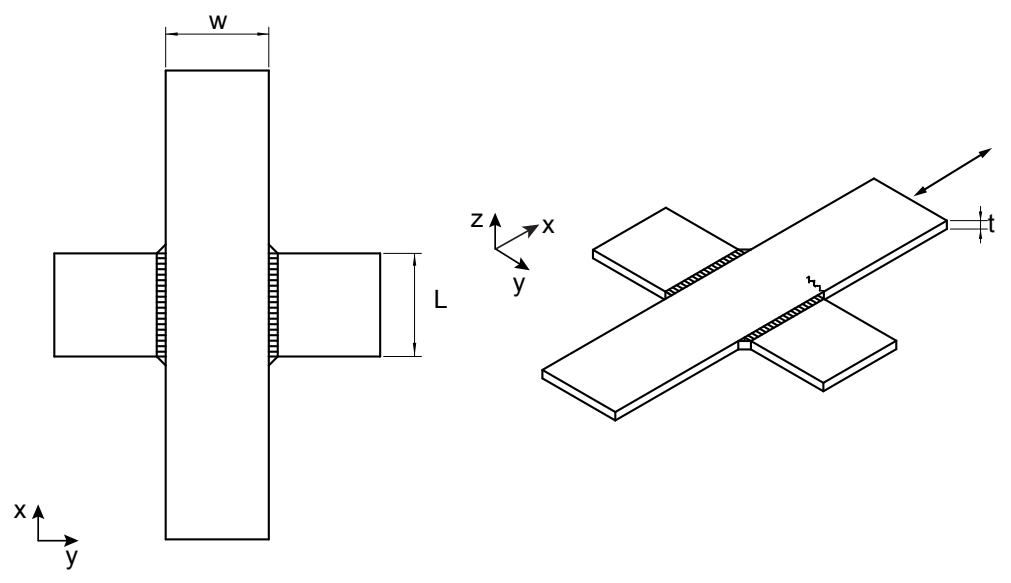

Figure A.17: In-plane gusset detail
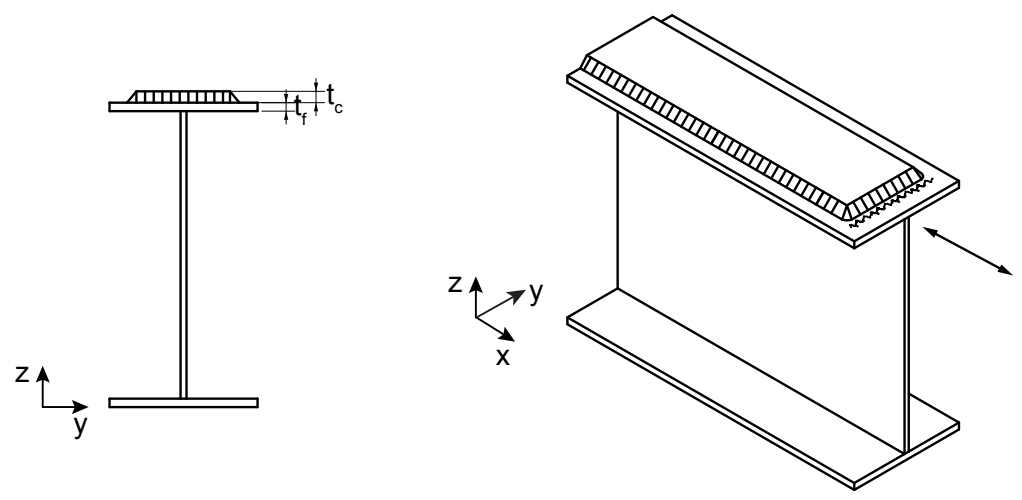

Figure A.18: Cover plate detail 


\begin{tabular}{|c|c|c|c|c|}
\hline Test series & $\mathrm{S}[\mathrm{MPa}]$ & $\mathrm{N}$ & $\delta_{i}$ & failure location \\
\hline & 80 & 1922000 & 1 & weld toe \\
\hline & 80 & 1810000 & 1 & weld toe \\
\hline Hirt (1975) & 120 & 514000 & 1 & weld toe \\
\hline \multirow[t]{3}{*}{$\mathrm{L}=100 \mathrm{~mm}$} & 120 & 361000 & 1 & weld toe \\
\hline & 160 & 210000 & 1 & weld toe \\
\hline & 160 & 199000 & 1 & weld toe \\
\hline \multirow[t]{5}{*}{ ICOM $(2015), \mathrm{L}=150 \mathrm{~mm}$} & 50 & 3800000 & 1 & weld toe \\
\hline & 140 & 528000 & 1 & weld toe \\
\hline & 140 & 252000 & 1 & weld toe \\
\hline & 140 & 272000 & 1 & weld toe \\
\hline & 100 & 826000 & 1 & weld toe \\
\hline Kondo (2002) & 80 & 1409000 & 1 & weld toe \\
\hline \multirow[t]{10}{*}{$\mathrm{L}=100 \mathrm{~mm}$} & 80 & 1431000 & 1 & weld toe \\
\hline & 60 & 16142000 & 0 & - \\
\hline & 60 & 5492000 & 1 & weld toe \\
\hline & 60 & 5144000 & 1 & weld toe \\
\hline & 55 & 4049000 & 1 & weld toe \\
\hline & 100 & 387000 & 1 & weld toe \\
\hline & 100 & 561000 & 1 & weld toe \\
\hline & 100 & 721000 & 1 & weld toe \\
\hline & 100 & 787000 & 1 & weld toe \\
\hline & 66 & 1000000 & 1 & weld toe \\
\hline & 66 & 1430000 & 1 & weld toe \\
\hline \multirow{6}{*}{$\mathrm{L}=150 \mathrm{~mm}$} & 66 & 1710000 & 1 & weld toe \\
\hline & 66 & 1730000 & 1 & weld toe \\
\hline & 33 & 13430000 & 0 & - \\
\hline & 33 & 16600000 & 0 & - \\
\hline & 33 & 18630000 & 0 & - \\
\hline & 33 & 23420000 & 0 & - \\
\hline
\end{tabular}

Table A.12: In-plane gusset, CA experimental data-set. $\delta_{i}$ is a binary variable which is equal to 1 for a failure and to 0 for a run-out 


\begin{tabular}{rrrrc} 
Test series & $\mathrm{S}[\mathrm{MPa}]$ & $\mathrm{N}$ & $\delta_{i}$ & failure location \\
\hline 27.6 & 100000000 & 0 & - \\
27.6 & 100000000 & 0 & - \\
27.6 & 100000000 & 0 & - \\
27.6 & 100000000 & 0 & - \\
31.7 & 100000000 & 0 & - \\
31.7 & 100000000 & 0 & - \\
31.7 & 100000000 & 0 & - \\
31.7 & 100000000 & 0 & - \\
32.4 & 34930000 & 1 & weld toe \\
& 32.4 & 37714000 & 1 & weld toe \\
34.5 & 16613000 & 1 & weld toe \\
Fisher (1977) & 34.5 & 32506000 & 1 & weld toe \\
34.5 & 8451000 & 1 & weld toe \\
34.5 & 47293000 & 1 & weld toe \\
34.5 & 89314000 & 0 & - \\
34.5 & 89314000 & 0 & - \\
41.4 & 11418000 & 1 & weld toe \\
41.4 & 12158000 & 1 & weld toe \\
41.4 & 4327000 & 1 & weld toe \\
41.4 & 12158000 & 1 & weld toe \\
41.4 & 100000000 & 0 & - \\
41.4 & 100000000 & 0 & - \\
55.2 & 2334000 & 1 & weld toe \\
55.2 & 5006000 & 1 & weld toe \\
55.2 & 4235000 & 1 & weld toe \\
55.2 & 1351300 & 1 & weld toe \\
\hline
\end{tabular}

Table A.13: Cover plate, CA experimental data-set. $\delta_{i}$ is a binary variable which is equal to 1 for a failure and to 0 for a run-out 


\begin{tabular}{c|r|r|r|r|r|r|r|r|r}
$\mathrm{S}_{\mathrm{i}}[\mathrm{MPa}]$ & \multicolumn{2}{|c|}{$\mathrm{n}_{\mathrm{i}}$} \\
\hline 30 & & & 2700 & 1560 & 12500 & 6400 & 2700 & $64 \cdot 10^{3}$ & $64 \cdot 10^{3}$ \\
40 & & 3278 & 1383 & 800 & & & & $33 \cdot 10^{3}$ & \\
50 & 3701 & 1896 & 800 & & & & 800 & & \\
60 & & & & & & & & & \\
70 & 1562 & 800 & & & & 800 & & $8 \cdot 10^{3}$ & $8 \cdot 10^{3}$ \\
80 & & & & & & & & & \\
90 & 800 & & & & 800 & & & & \\
100 & 1.11 & 1.61 & 4.61 & $>40.0$ & 6.78 & 13.2 & 9.52 & 3.17 & 15.08 \\
& 1.18 & 2.43 & 4.73 & 10.4 & 9.64 & 9.15 & $>22.1$ & 3.65 & \\
$\mathrm{n}_{\text {fail }} \cdot 10^{6} \rightarrow$ & & & & 14.0 & & & 16.1 & 3.67 & \\
& & & & & & & $>29.6$ & & \\
\end{tabular}

Table B.14: In-plane gusset, VA experimental data-set. The column $S_{i}$ represents the stress ranges of the loading spectrum. The columns $n_{i}$ represent the number of cycles associated to the stress ranges of the first column; each column of $n_{i}$ represents a different spectrum. In the lower part of the table, the number of cycles to failure, $n_{\text {fail }}$, are presented; the values which are presented in the same column were obtained under the same loading spectrum. The symbol $>$ is used to indicate a run-out

\section{Appendix B. VA experimental data-sets}

In this section the two analysed VA experimental data-sets are presented. The loading spectra, the number of cycles to the end of the test and the test output are presented in Tables B.14 and B.15, whose captions explains how to read the Table. 


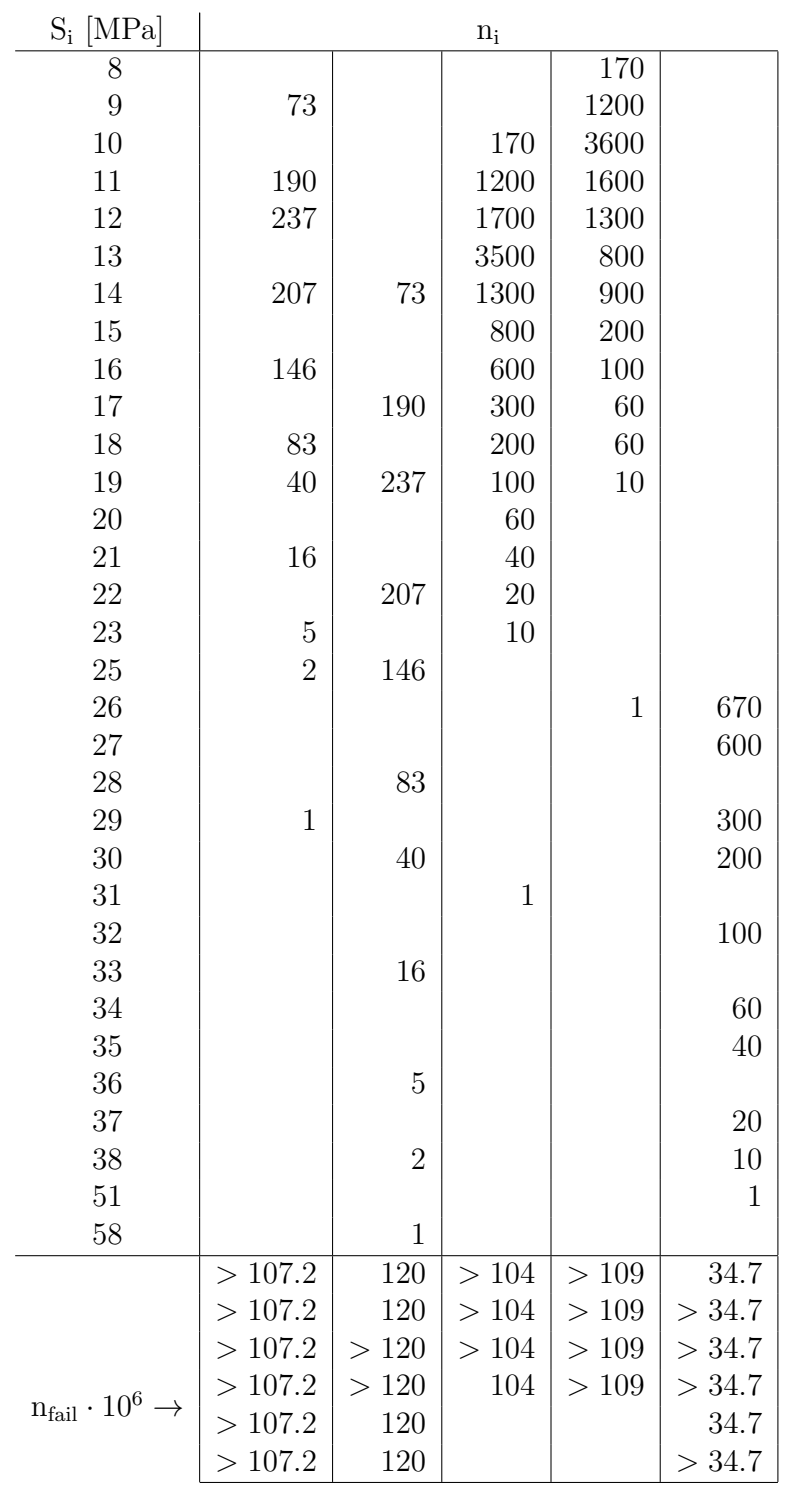

Table B.15: Cover plate, VA experimental data-set. The column $S_{i}$ represents the stress ranges of the loading spectrum. The columns $n_{i}$ represent the number of cycles associated to the stress ranges of the first column; each column of $n_{i}$ represents a different spectrum. In the lower part of the table, the number of cycles to failure, $n_{\text {fail }}$, are presented; the values which are presented in the same column were obtained under the same loading spectrum. The symbol $>$ is used to indicate a run-out 
[1] E. Haibach, Modifizierte lineare Schadensakkumulations-Hypothese zur Berücksichtigung des Dauerfestigkeitsabfalls mit fortschreitender Schädigung. , Juli 1970, TM Nr. 50/70., Laboratorium für Betriebsfestigkeit, 1970.

[2] S. G, A. Hobbacher, A. Nussbaumer, J. Stotzel, D. Schafer, Commentary to Eurocode 3. Part 1-9 - Fatigue (2014).

[3] J. Spindel, E. Haibach, The method of maximum likelihood applied to the statistical analysis of fatigue data, International Journal of Fatigue 1 (April) (1979) 81-88.

[4] W. Nelson, Fitting of Fatigue Curves with Nonconstant Standard Deviation to Data with Runouts, Journal of Testing and Evaluation 12 (1984) 69-77.

[5] F. G. Pascual, W. Q. Meeker, Estimating Fatigue Curves With the Random Fatigue-Limit Model, Technometrics.

[6] T. Lassen, P. Darcis, N. Recho, Fatigue Behavior of Welded Joints Part 1 - Statistical Methods for, Supplement to the Welding Journal (December) (2005) 183-187.

[7] Joint Committee Structural Safety, JCSS Probabilistic Model Code: Resistance Models (2013).

[8] C. Sonsino, Course of SN-curves especially in the high-cycle fatigue regime with regard to component design and safety, International Journal of Fatigue 29 (12) (2007) 2246-2258.

[9] European Committee for Standardization, Eurocode 3: Design of steel structures - Part 1-9: Fatigue (2005).

[10] A. Hobbacher, IIW document XIII-2460-13/XV-1440-13 Recommendations for fatigue design of welded joints and components (2013).

[11] E. Haibach, The allowable stresses under variable amplitude loading of welded joints, in: The Welding Institute (Ed.), Proceedings of the conference on fatigue of welded structures, Abington, Cambridge, 1971, pp. 328-346. 
[12] AASHTO, AASHTO LFRD Bridge Design Specifications Customary U.S. Units (2012).

[13] J. W. Fisher, A. Nussbaumer, P. B. Keating, B. T. Yen, Resistance of Welded Details Under Variable Amplitude Long-Life Fatigue Loading, Tech. rep., National Cooperative Highway Research Program, Bethlehem, Pennsylvania (1993).

[14] G. Marquis, Long life spectrum fatigue of carbon and stainless steel, Fatigue \& Fracture of Engineering Materials and Structures 19 (6) (1996) $739-753$.

[15] T. Gurney, Fatigue of welded structures, 2nd Edition, Cambridge University Press, Abington, Cambridge, UK, 1979.

[16] Y. Zhang, S. Maddox, Investigation of fatigue damage to welded joints under variable amplitude loading spectra, International Journal of Fatigue 31 (1) (2009) 138-152.

[17] P. Albrecht, H. Lu, K. Jung, H. J. Liu, J. G. Cheng, Long life variablamplitude fatigue strength of welded steel bridge details, Tech. rep., Federal Highway Administration, Washington DC, USA (1994).

[18] M. A. Miner, Cumulative damage in fatigue, Journal of Applied Mechanics 12 (1945) 159-164.

[19] C. G. Schilling, K. Klippenstein, J. M. Barsom, G. T. Blake, Fatigue of welded steel bridge members under variable amplitude loadings, NCHRP Final Report 12, Tech. rep., Bethlehem, Pennsylvania (1975).

[20] Welding Institute, Fatigue performance of welded high strength steels: a compendium of reports from a sponsored research programme, Report series, Welding Institute, Cambridge, UK, 1974.

[21] S. Berge, Residual stress and stress interaction in fatigue testing of welded joints. Report SK/R55, Tech. rep., University of Trondheim, Trondheim (1981).

[22] P. Albrecht, K. Yamada, Simulation of service fatigue loads for short span highway bridges, in: ASTM STP 671, Philadelphia, USA, 1979. 
[23] D. Benoit, H. P. Lieurade, M. Truchon, Fatigue behaviour under programmed loading of welded cruciform and butt joints in steel E355. ECSC Report 6210, Tech. rep., CETIS, Senlis, France (1977).

[24] E. Gassner, Endurance stresses and fatigue life of a mild steel weld under different types of load spectra, Welding Research Abroad 11 (4) (1965) $23-477$.

[25] C. A. Baptista, L. D'Angelo, Experimental fatigue tests of in-plane welded gusset joints. EPFL-REPORT-213745, Tech. rep., Ècole Polytechnique Fédérale de Lausanne, Lausanne (2015).

[26] R. B. Millar, Maximum Likelihood Estimation and Inference: With Examples in R, SAS ans ADMB, Wiley, Chichester, UK, 2011.

[27] M. Vormwald, T. Seeger, Consideration of fatigue damage below the endurance limit in life predictions for variable amplitude loadings, in: Proceedings of the Fourth International Conference on Fatigue and Fatigue Thresholds, Honolulu, Hawaii, pp. 517-523. 